\title{
Optical 4D oxygen mapping of microperfused tissue models with tunable in vivo-like 3D oxygen microenvironments
}

\author{
Milan Finn Wesseler, Mathias Nørbæk Johansen, Aysel Kızıltay, Kim I. Mortensen, and
} Niels B. Larsen*

Department of Health Technology, DTU Health Tech, Technical University of Denmark, $2800 \mathrm{Kgs.}$ Lyngby, Denmark

E-mail:nibl@dtu.dk

Keywords: Oxygen analysis; microsensor; in vitro; tissue culture; organ models; phosphorescence lifetime microscopy (PLIM);

\begin{abstract}
Sufficient and controllable oxygen supply is essential for in vitro 3D cell and tissue culture at high cell densities, which calls for volumetric in situ oxygen analysis methods to quantitatively assess the oxygen distribution. This paper presents a general approach for accurate and precise non-contact 3D mapping of oxygen tension in high cell-density cultures via embedded commercially available oxygen microsensor beads read out by confocal phosphorescence lifetime microscopy (PLIM). Optimal acquisition conditions and data analysis procedures are established and implemented in a publicly available software package. The versatility of the established method is first demonstrated in modelassisted fluidic design of microperfused 3D printed hydrogel culture chips with the aim of full culture oxygenation, and subsequently for monitoring and maintenance of physiologically relevant spatial and temporal oxygen gradients in the 3D printed chips controlled by static or dynamic flow conditions during 3D culture.
\end{abstract}

\section{Introduction}

True in vitro 3D culture is limited by the ability to supply the right amount of oxygen to cells for extended time periods ${ }^{1,2}$ and to spatially control oxygen concentrations in the culture volume to reproduce in vivo conditions. ${ }^{3}$ Engineered microperfusion systems may overcome such limitations, as demonstrated in our previous work on 3D printing microperfusable cell culture environments, ${ }^{4}$ given the availability of solutions to accurately monitor oxygen levels in a time-dependent manner in 3D.

Non-invasive oxygen mapping using optical readout of oxygen-dependent fluorescence and phosphorescence of added dissolved sensor species ${ }^{5}$ have been widely exploited for in vivo ${ }^{6,7}$ and in vitro $^{8,9}$ analysis at cellular to tissue length scales. The process generally involves quenching of an excited molecular state by oxygen, resulting in the generation of cytotoxic singlet oxygen species. ${ }^{5}$ This may limit the allowable concentration of dissolved oxygen sensor species for continuous cell culture studies. Embedding sensor species and singlet oxygen scavenging species ${ }^{10}$ in microbeads (sensor beads) made from a water-impermeable matrix such as poly(dimethylsiloxane) (PDMS) ${ }^{11-13}$ or polystyrene (PS) ${ }^{14,15}$ largely eliminates issues of cytotoxicity. This permits the use of higher sensor species concentrations to increase the signal-to-noise ratio for faster data acquisition as well as reducing toxicity during long-term culture.

Sub-micron sized sensor beads for intracellular oxygen mapping in cell cultures were reported more than two decades ago. ${ }^{16} \mathrm{At}$ length scales of tissues, sensor beads have most commonly been employed for measuring in-plane oxygen variations, i.e., either at an interface or with observations 
being projected onto a plane. ${ }^{12,13,15,17} \mathrm{~A}$ recent report used custom-synthetized sensor beads to map the oxygen distribution in static cell cultures with some vertical detail. ${ }^{18}$ However, the authors did not target high cell density cultures and had less focus on determining the oxygen concentration 3D with high accuracy. To the best of our knowledge, dispersed microbeads have not previously been used as a tool for tracking spatiotemporal changes in 3D cultures depending on microperfusion geometry and conditions. In the present work, we explore the use of commercially available sensor beads evenly dispersed in cell-embedding hydrogels to accurately monitor and engineer oxygen concentrations generated by microfluidic channel arrays manufactured in oxygen-permeable materials by stereolithographic 3D printing. The aim is to track spatiotemporal variations with minute scale time resolution in a setup compatible with week-long longitudinal 3D cell culture studies.

The oxygen-dependent quenching of excited sensor species can be detected by ( $i$ ) a reduction in the number of photons emitted by the sensor species, by (ii) a phase change between a periodically varying excitation and emission intensity, or by (iii) measurement of the oxygen-dependent shortening of the intensity decay profile after a pulsed excitation of the sensor species. Our focus is on achieving the most accurate and precise conversion from quenching of excited sensor species to dissolved oxygen concentration in 3D measurements. The third detection scheme has the highest robustness to the strong intensity variations inherent to deep optical probing into high cell density cultures. Thus, the chosen analysis system relies on spatially resolved measurements of timedependent phosphorescence decay profiles using Time-Correlated Single Photon Counting (TCSPC). ${ }^{19}$ This is experimentally implemented using confocal phosphorescence lifetime microscopy (PLIM). ${ }^{20}$

Additionally, we report a quantitative comparison of key mathematical modeling methods of the phosphorescence decay to reach the best accuracy and precision for a wide range of phosphorescence intensity levels as observed during 3D confocal visualization of the sensor beads. We have integrated the optimal fitting and conversion method in a MATLAB-based (MathWorks) automated analysis and visualization software package. The package is publicly available on request as a turn-key 3D oxygen mapping solution for laboratories with access to a custom-built or commercially available PLIM hardware platform such as the one used in this work. We show that the method is equally applicable to static and dynamic flow systems, and that 3D cell cultures even far from physiological relevant cell densities may experience strongly hypoxic or fully anoxic conditions in the absence of densely spaced microperfusion networks.

\section{Results and Discussion}

We first established a reproducible general workflow for quantitative mapping of oxygen concentrations in 3D cell culture environments. The process (Fig. 1) uses commercially available oxygen sensor beads ( $\varnothing 50 \mu \mathrm{m}, \mathrm{CPOx}$-Red, Colibri Photonics) ${ }^{21}$ that are mixed with cells, in the presence or absence of an embedding hydrogel matrix (Fig. 1a), prior to seeding into a culture volume (Fig. 1b). The local oxygen concentration is probed using a commercial confocal phosphorescence life-time microscope (PLIM, Becker \& HickI DCS-120 mounted on a Zeiss Z1 inverted microscope) programmed to record phosphorescence decay profiles across the entire culture volume (Fig. 1C). This permits accurate mapping of oxygen concentrations in 3D with subminute scale time resolution (4D mapping), given that reliable analysis procedures can be established for decay profiles with limited total signal intensity and signal-to-noise ratios. 


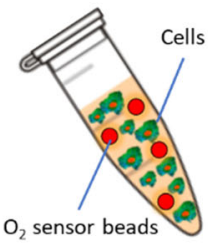

b)

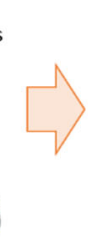

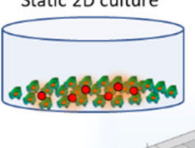
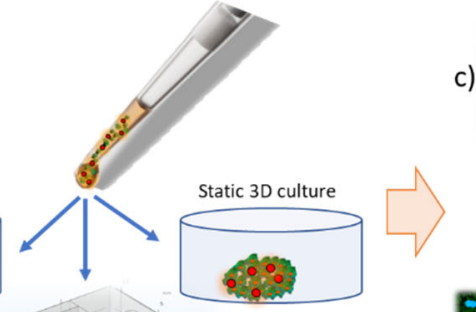

c)

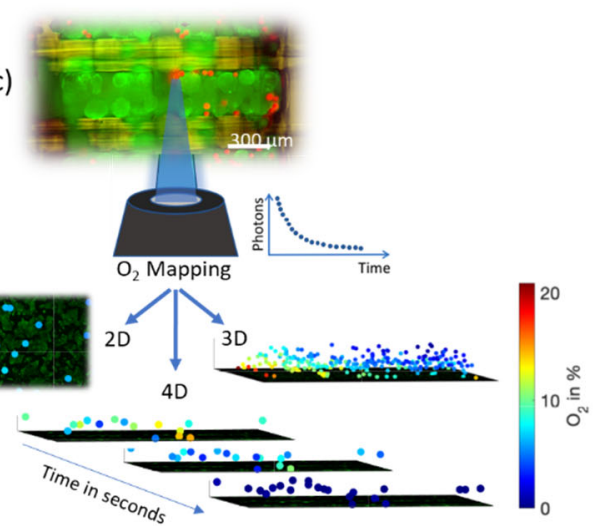

Fig. 1 (a) The general analysis procedure starts by mixing cells and oxygen sensor beads $(\varnothing 50 \mu \mathrm{m})$ and optionally an embedding hydrogel. (b) The cell mixture is dispensed into the targeted culture format, here illustrating conventional 2D static culture, 3D static culture, and 3D dynamic culture within a chip supporting microperfusion. (c) Confocal phosphorescence lifetime microscopy (see main text) mapping of the oxygendependent phosphorescence decay profiles of the embedded sensor beads can be converted to calibrated oxygen tension maps in 2D, 3D, or 4D.

\section{Oxygen mapping in static 3D cell cultures}

We initially studied hydrogel-embedded cell cultures under static medium conditions to establish the analysis process in a simpler 3D environment that still recapitulates the challenges of widely varying sensor bead signal intensities resulting from differences in probing depths. Fig. 2 shows the model system employed, where cells at high seeding density and sensor beads are suspended in a nearly hemispherical droplet of photo-crosslinkable gelatin methacryloyl (GelMA) hydrogel ${ }^{22}$ deposited at a well bottom of a 96-well plate. The GelMA solution is subsequently photo-crosslinked into an embedding hydrogel that maintains the cells' and sensor beads' 3D locations at the time of light exposure. The cell-containing droplet is either covered directly with medium (Fig. 2a) or first covered with a layer of cell-free GelMA hydrogel before addition of medium (Fig. $\mathbf{2 b}$ ). The addition of a layer of cell-free hydrogel inhibits convective medium flow at the perimeter of the cell-laden hydrogel droplet, expectedly leading to strongly reduced oxygen replenishment within the droplet volume. A similar configuration was recently investigated by Wilson et al. using approximately 10 times lower cell densities. ${ }^{18}$ Confocal PLIM analysis of the two system configurations after 4 days of culture is overlaid on confocal fluorescence microscopy of live-cell staining (calcein AM) obtained in parallel (Fig. 2c and d). Each embedded and analyzed sensor bead is represented by a filled circle color-coded with the determined oxygen concentration in lateral $(X / Y)$ and radial cross-sectional (R/Z) projections of the combined confocal PLIM/fluorescence stacks. The vertical position of each plotted sensor bead is estimated from the optically "stretched" confocal stacks. The hemispherical hydrogel model was also employed for assessing the accuracy by which the vertical position can be predicted, see Electronic Supplementary Information (ESI) Section 1 and Fig. S1. 

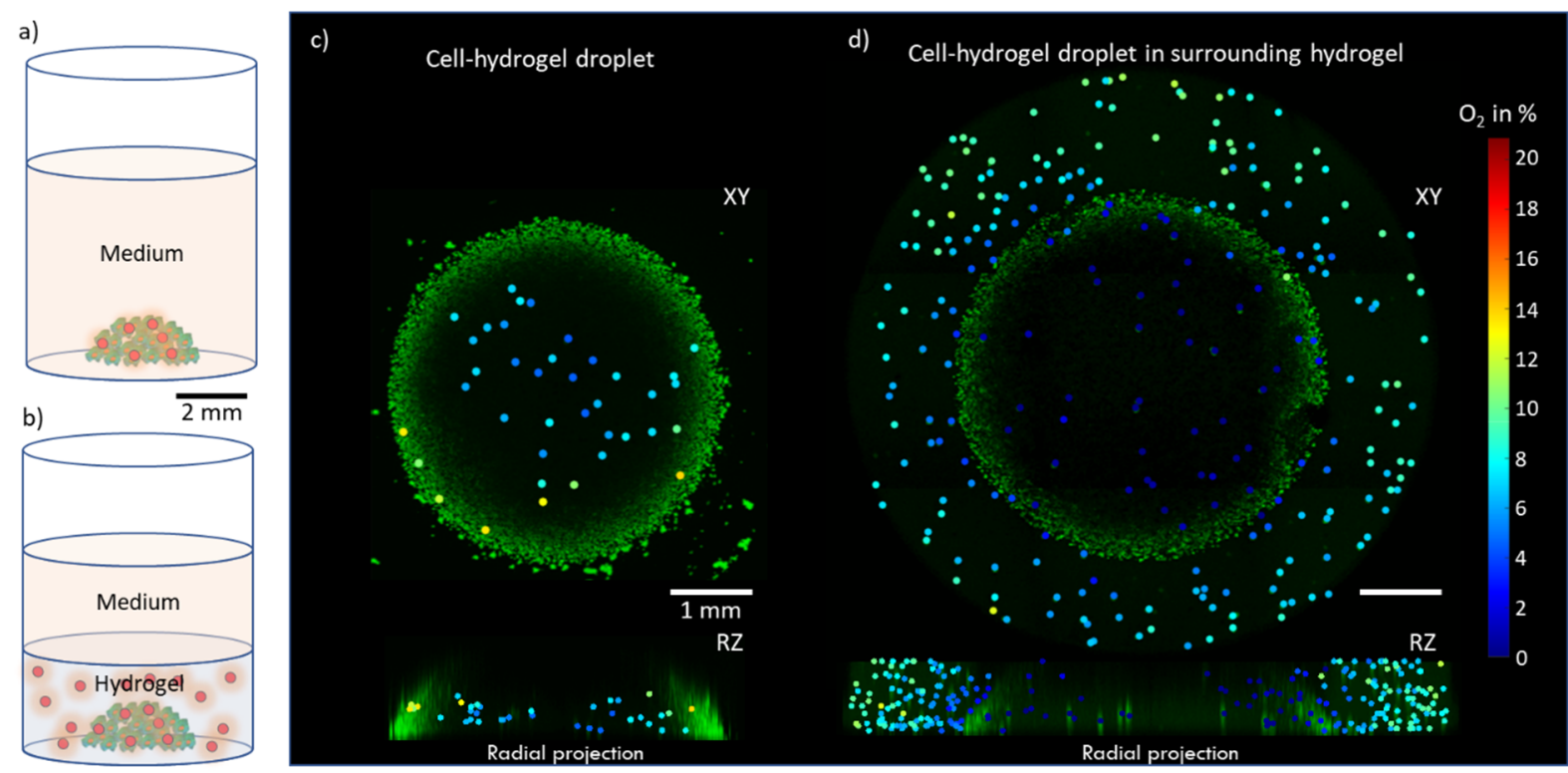

Fig. 2 3D oxygen mapping of hydrogel-embedded high-density cell cultures under static culture conditions in wells of a 96-well plate, either (a) as a droplet in medium (cells and sensor beads shown in green and red, respectively) or (b) as a cell-laden droplet surrounded by a cell-free hydrogel covered with medium. (c and d) Confocal PLIM imaging of the embedded oxygen sensor beads and subsequent conversion to the corresponding local oxygen concentration is shown as color coded concentrations overlaid with green fluorescence from Calcein AM staining of metabolically active cells in lateral (XY) and radial (RZ) projections. Hep $\mathrm{G} 2$ cells at $20 \times 10^{6}$ cells $/ \mathrm{mL}$ embedded in a hydrogel of $7.5 \% \mathrm{w} / \mathrm{v}$ GelMA in medium without or with a second added hydrogel volume of $7.5 \%$ GelMA in medium.

The cell-laden droplet surrounded by culture medium shows gradually decreasing oxygen concentrations from its perimeter to its center due to oxygen being consumed by the embedded metabolically active Hep $\mathrm{G} 2$ hepatocytes (Fig. 2c). Still, the oxygen concentration in the droplet center remains at a level of 6-8\%, which is sufficient to support their normal metabolism. In contrast, an equivalent droplet surrounded by a cell-free hydrogel exhibits nearly anoxic conditions in its entire volume except for the outermost $100 \mu \mathrm{m}$ (Fig. 2d). The observed difference between the two configurations illustrates the importance of accurately describing the type of nominally static culture conditions employed. Interestingly, the intended green fluorescent staining of live cells by calcium AM does not correlate well with the oxygen concentration profiles across the beads. We assume this is caused by very fast uptake of the added calcium AM just below the hydrogel droplet surface due to the very high cell density employed in this study. This observation supports the need for accurate volumetric oxygen mapping to truly describe the metabolic environment at cell densities approaching those of organ tissues.

\section{Establishing an accurate and precise oxygen mapping algorithm}

Fig. $2 \mathrm{c}$ and $\mathrm{d}$ show that consistent 3D oxygen concentration measurements can be performed for depths of $\geq 1 \mathrm{~mm}$ into high cell density cultures using our developed analysis process. This process consists of (a) parallel volumetric acquisition of sample fluorescence and phosphorescence, (b) automated identification and pooling of acquired phosphorescence decay profiles from equivalent sensing locations within each bead to increase the signal-to-noise ratio; (c) correction for common systematic errors due to limitations of the acquisition hardware; and (d) robust fitting of the pooled decay profile of each bead to extract the corresponding oxygen concentration.

The first three process steps are detailed in Fig. 3. Individual voxels in a confocal stack are sequentially analyzed to produce a volumetric map of sample fluorescence and sensor bead 
phosphorescence decay profiles (Fig. 3a). The time-dependent photon flux integrated over the area (voxels) of a representative sensor bead in a single acquisition plane is shown in Fig. 3b. Each voxel is first illuminated by the pulsed excitation laser for a sub-100 $\mu$ s period ("Laser on", Fig. 3b) to generate a steady state population of excited sensor molecules. During this excitation phase, fluorescence data (ns-scale decay times) are acquired between each excitation light pulse $(80 \mathrm{MHz}$ repeat rate), which enables parallel visualization of fluorescently labeled cells and sensor beads. In the subsequent "Laser off" period, only emission from triplet states of the sensor molecules contribute to the decay curve. Individual voxels of the phosphorescence confocal stack (Fig. 3c) typically emit $10-150$ photons per analysis cycle (Fig. 3d). This is insufficient for high-quality fitting of the decay curve profile. The developed software package sorts through the confocal stack to identify the centroid and extent of each bead (Fig. 3e) and sums the photons from voxels of each bead into a combined decay curve with high signal-to-noise ratio (Fig. 3f, red curve). The sorting procedure and the experimental support for equivalence of decay curves of all voxels in a bead are detailed in ESI sections 2.1 and 2.2. As the final step, the combined decay curve is corrected for any systematic hardware-dependent deviations (Fig. 3f, blue curve), including the single-photon counter deadtime leading to an underestimation of the photon count at high photon fluxes (so-called sensor saturation).

a)

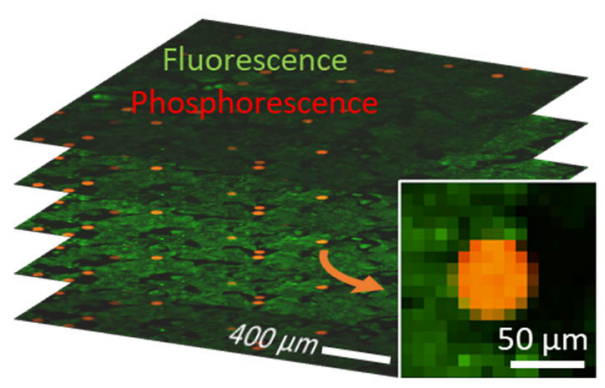

c)

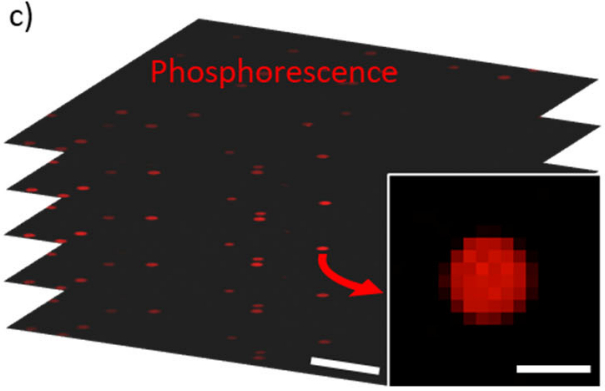

e)

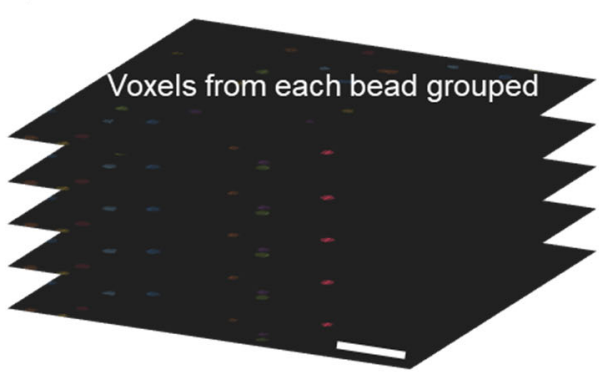

b)

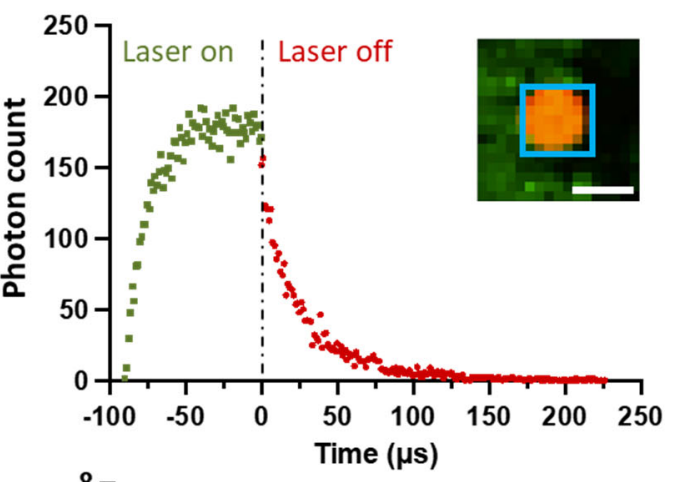

d)

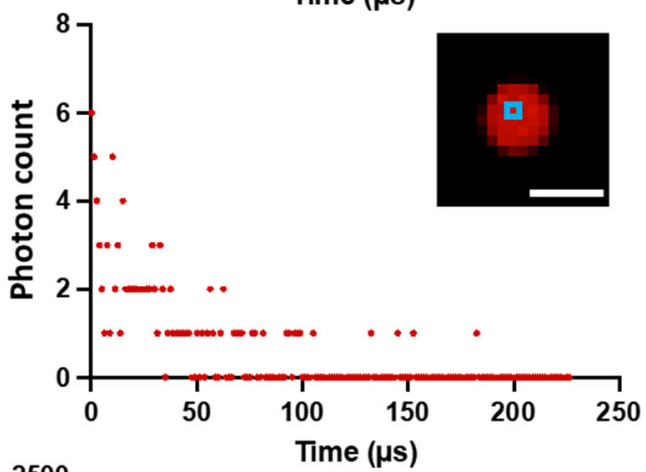

f)

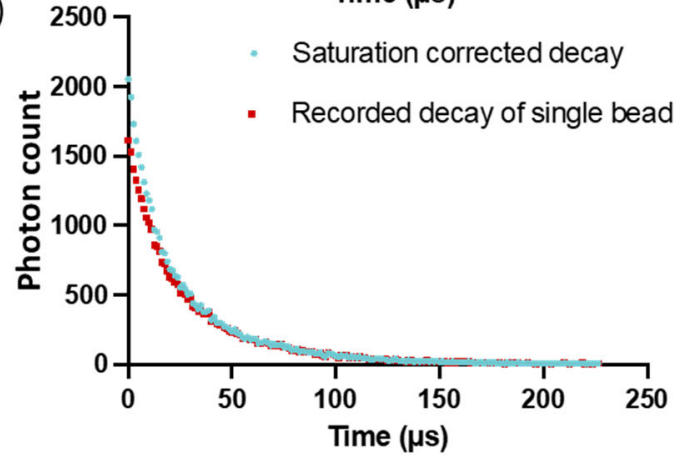

Fig. 3 (a) Combined confocal stacks of fluorescence and phosphorescence shows the distribution of sensor beads (orange; both fluorescence and phosphorescence) within a live-stained (green; fluorescence) 3D cell 
culture. (b) Phosphorescence decay profile maps are recorded through excitation ("Laser on") at each voxel location followed by single photon counting of the intensity decay during an excitation free period ("Laser off"). The graph shows the aggregate photon count over the spatial extent of a single bead in one image plane. (c) Confocal maps of the time-integrated phosphorescence photon counts of (d) the small number of photons in each pixel's decay curve are used for (e) identifying the volumetric extent of all beads to allow for ( $f$ ) summation of decay curves over each entire bead to improve the signal-to-noise ratio before final correction for instrument-dependent contributions (detector saturation).

Oxygen-dependent fluorescence and phosphorescence decay curves have been investigated extensively. ${ }^{23}$ The proposed models of decay profiles from phosphorescent species immobilized in a diffusion-limiting matrix, such as polystyrene in the current work, can be divided in two main classes. The first class formally describes individual species to be either fully accessible or fully inaccessible to the dissolved oxygen. ${ }^{24}$ The expected decay profile will be a biexponential, with one long constant decay time for inaccessible species and a shorter variable decay time for accessible species. Full accessibility or inaccessibility to all sensor species are special cases of this first class, where a single exponential with variable decay time can fully describe the decay profile. The second class of model assumes that all species will be accessible to the dissolved oxygen to varying degrees, resulting in a continuum of decay times. This can mathematically be modeled by a stretched exponential with a stretching factor $\beta,{ }^{25}$ where $\beta=1$ is the special case of a single exponential decay curve.

Phosphorescence decay profiles have most commonly been fitted using a non-linear least squares (NLLS) approach. ${ }^{23}$ NLLS assumes a normal distribution of the photon counts within each of the analyzed discrete time bins, which is a good approximation at a high number of binned photon counts. However, the low photon counts expected for the required short acquisition times per bead are predicted to follow a Poisson distribution instead of a normal distribution. The change in distribution can be accounted for by generalizing the fitting procedure to Maximum Likelihood Estimation (MLE), ${ }^{26-28}$ having NLLS as a limiting case for large binned photon numbers. We performed an extensive comparison of the two fitting approaches, described in detail in the ESI section 2.3. Our main conclusion is that MLE indeed produces a statistically better curve fit in the low-intensity tail part of the decay curves, but that the difference in fitted decay times between NLLS and MLE generally is small. MLE never performs worse than NLLS and does not incur significant computational overhead, so we continued with MLE as our fitting approach.

We next compared the two classes of decay profile models, with the inclusion of a single exponential decay as a special case of both model classes. The underlying experimental data was acquired on sensor beads embedded in a GelMA hydrogel placed within a gas-tight chamber. The chamber ambient oxygen concentration was controlled by combinations of air and nitrogen supplied by mass flow controllers. The special case of completely oxygen-free conditions was confirmed by the dissolution of sodium sulfite to scavenge any dissolved oxygen remaining in solution below the nominally oxygen-free ambient. We employed the Akaike information criterion (AIC) ${ }^{29}$ as measure of the obtained quality of fit penalized by the required number of fitting parameters (see Materials and Methods and ESI section 2.4). Fig. 4a and b compare the fitting results for the minimum and maximum concentrations of $0 \%$ and $20.9 \%$ oxygen. At $0 \%$ oxygen, all three models fit the data with visually indistinguishable quality. This is also expected given that the single exponential emerges as a special case of the biexponential for fully inaccessible sensor species and of the stretched exponential for $\beta=1$. At the other extreme, $21 \%$ oxygen, the fitted single exponential overestimates the photon counts at short and long decay times while underestimating the intensity at intermediate decay times. This is clearly visible in the inset showing the photon count on a logarithmic scale. The stretched exponential (fitted stretching factors displayed in Fig. 4c) and the biexponential provided fits of comparable visual quality and residuals. Yet, the weighted AIC scores 
for all three models, with higher being better, showed that the biexponential is uniformly the best choice across all tested oxygen concentrations (Fig. 4d). The fitted oxygen-decay time calibration curves and corresponding Stern-Volmer plots, ${ }^{30}$ plots for all three models are shown in Fig. $4 \mathrm{e}$ and f. The calibration based on a biexponential is used going forward.

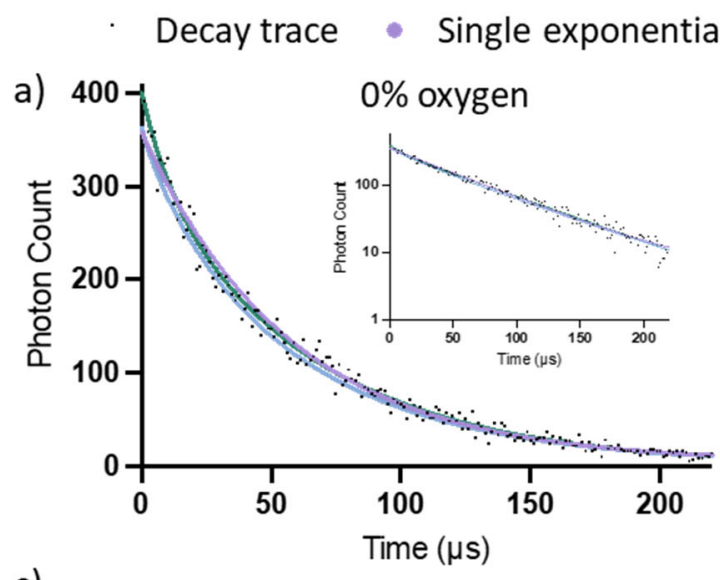

- Stretched exponential $\Delta$ Biexponential
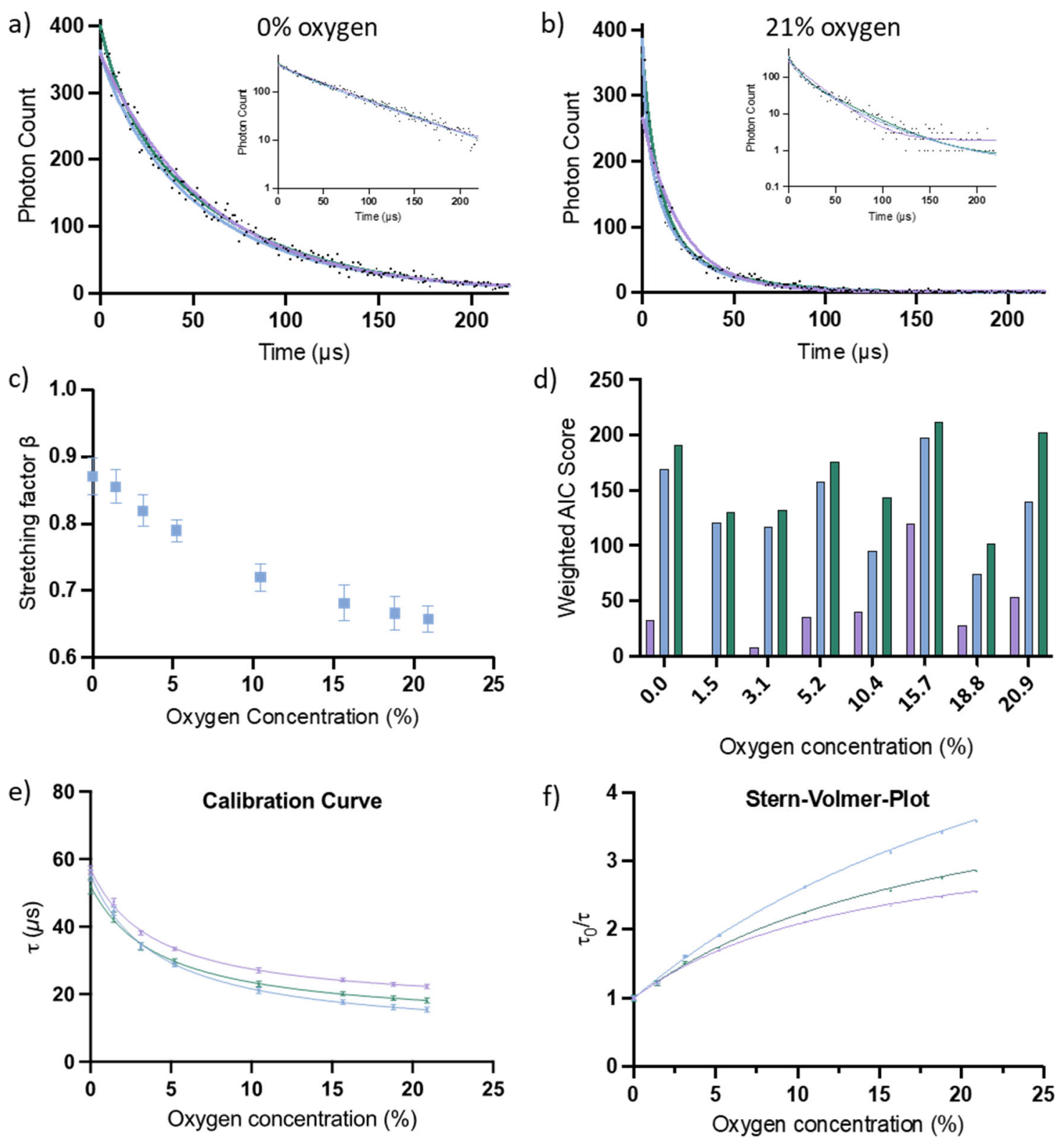

Fig. 4 ( $a$ and b) Saturation-corrected decay traces for 0\% (a) and 21\% (b) oxygen tension, with insets showing the photon counts on a logarithmic scale. Solid lines show fits using models of, respectively, a single exponential decay (purple), a stretched exponential decay (teal), and a biexponential decay (green). (c) Optimal stretching factors (mean +/- s.d.) for the stretched exponential over beads as a function of oxygen tension. (d) Weighted AIC scores (see main text, higher is better) for the three models applied to $45-173$ beads. This quantifies the quality of fits penalized by the number of parameters in each model. (e and f) Calibration curve and Stern-Volmer plot for converting decay lifetime into oxygen concentration based on lifetimes obtained by the three different models (mean +/- s.e.m., 3-7 independent experiments, 45-173 sensor beads).

As discussed, the signal-to-noise ratio affects the quality of the fit and thereby the uncertainty on the estimated decay time. The automated analysis procedure discards beads with too few detected 
photons to give a precise oxygen concentration estimate. We experimentally found that decay curves from $<600$ photons resulted in strongly increasing standard deviations of the oxygen concentration estimate (ESI section 2.6 and Fig. S6). A conservative threshold of $\geq 1000$ photons per sensor bead for inclusion in 3D maps was generally employed.

We used standard 2D high cell density cultures in microtiter plates as a simple reference system to investigate the homogeneity of the measured oxygen concentrations in a real in vitro environment. Fig. 5 compares the oxygen concentration right above confluent cell monolayers in a 96 well plate with overlaying medium thickness of $1.5 \mathrm{~mm}(50 \mu \mathrm{L}), 3.0 \mathrm{~mm}(100 \mu \mathrm{L})$, and $6.0 \mathrm{~mm}(200 \mu \mathrm{L})$, respectively. The color-coded sensor beads reveal a strong decrease in oxygen tension with medium depth, from $18-19 \%$ at the smallest to $7-8 \%$ at the largest medium depth. This is a reminder that full oxygenation of dense 2D cell layers cannot be assumed. However, it may also be exploited as an experimental design parameter to achieve physoxic 2D culture conditions without active oxygen control in incubators with standard gas compositions ( $95 \%$ air, $\left.5 \% \mathrm{CO}_{2}\right)$. The observed variation in oxygen tension across the three sample surfaces is less than $1.5 \%$ (standard deviation of $1.5 \%$ for 50 $\mu \mathrm{L}, 1.3 \%$ for $100 \mu \mathrm{L}, 0.8 \%$ for $200 \mu \mathrm{L}$ ), which supports a high precision of the developed analysis procedure.
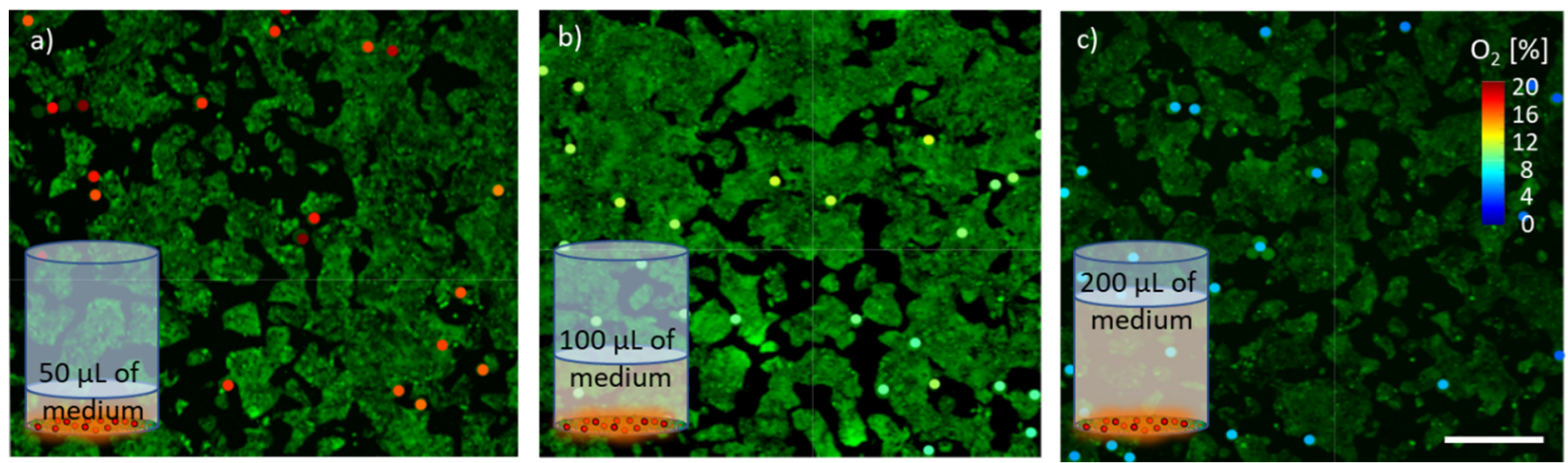

Fig. 5 Dense planar cultures, here in a 96-well microtiter plate, also experience significant oxygen depletion when increasing the amount of medium volume, from (a) $50 \mu \mathrm{L}$ ( $1.5 \mathrm{~mm}$ medium depth) through (b) $100 \mu \mathrm{L}$ $(3.0 \mathrm{~mm})$ to (c) $200 \mu \mathrm{L}(6.0 \mathrm{~mm})$, as revealed by sensor beads (color coded) sedimented on top of the cell layer. Hep G2 cells on day 4 after seeding at $30 \times 10^{3}$ cells/well; live staining using calcium AM (green). Scalebar 500 $\mu \mathrm{m}$.

\section{Microfluidic design supported by 3D oxygen mapping}

We hypothesized that our full 3D oxygen mapping method can be employed for functional assessment and optimization of microfluidic designs supporting oxygenation in perfused 3D cell culture systems. The first culture chip design incorporates a single fluidic channel traversing the bottom of a culture confinement (Fig. 6 and ESI Section 3). Monolithic chips including supply channels and access ports for fluidic connectors were 3D printed in an oxygen diffusion-open hydrogel material, poly(ethylene glycol) diacrylate $M_{n} 700$ Da (PEGDA 700), using the high-resolution stereolithography printing system and protocols reported in our recent work. ${ }^{4,31}$ The cell culture confinement was seeded with a suspension of Hep G2 cells $\left(5 \times 10^{6}\right.$ cells $\left./ \mathrm{mL}\right)$ and oxygen sensor beads in an aqueous solution of GelMA subsequently photo-crosslinked into a hydrogel. The perfusion channel was connected to an external peristaltic pump through the access ports, and the connected chip was fully immersed in cell culture medium in a 6-well microtiter plate. Culture proceeded in a custom-built microscope-compatible mini-incubator $\left(37^{\circ} \mathrm{C}, 5 \% \mathrm{CO}_{2}\right)$ using an average medium perfusion velocity of $3.2 \mathrm{~mm} / \mathrm{s}$ through the channel. The oxygen concentration distribution was analyzed on culture day 3 (approximately $56 \mathrm{~h}$ after seeding). Fig. $6 \mathrm{~b}$ and c show representative lateral and cross-sectional projections of all the analyzed sensor beads overlaid on the projected 
(green) fluorescence of live cells stained by perfusion of dissolved calcium AM for 90 min prior to visualization. Nearly anoxic conditions are found at distances greater than $500 \mu \mathrm{m}$ from the outer channel wall, which roughly correlates with the observed spatial decay of the live-stain fluorescence from the perfusion channel. Medium perfusion (advective flow), oxygen diffusion, and cellular oxygen consumption were modeled at steady state using finite element modeling (COMSOL, details in ESI Section 3) for a range of oxygen consumption rates. Fig. $\mathbf{6 d}$ shows the resulting calculated volumetric oxygen distribution as a cross-sectional view half-way down the fluidic channel, showing good agreement with the experimental results outside the fluidic channel in Fig. $6 \mathrm{c}$ for a modeled oxygen consumption rate (OCR) of $1.1 \times 10^{-16} \mathrm{~mol} \mathrm{cell}^{-1} \mathrm{~s}^{-1}$. This is in good agreement with previously reported values OCR values for Hep G2 of $0.8 \times 10^{-16}-1.1 \times 10^{-16} \mathrm{~mol} \mathrm{cell}^{-1} \mathrm{~s}^{-1} .^{32,33}$
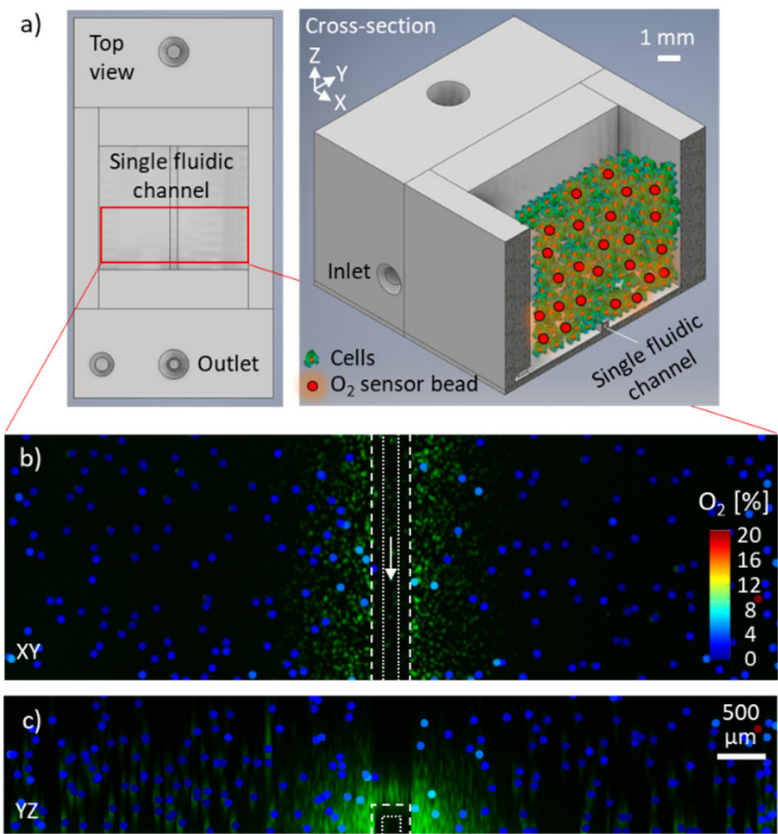

d)

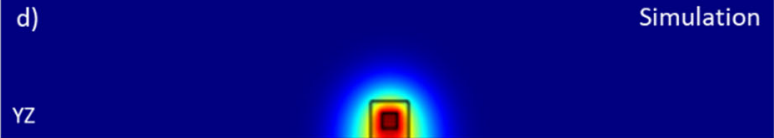

e)
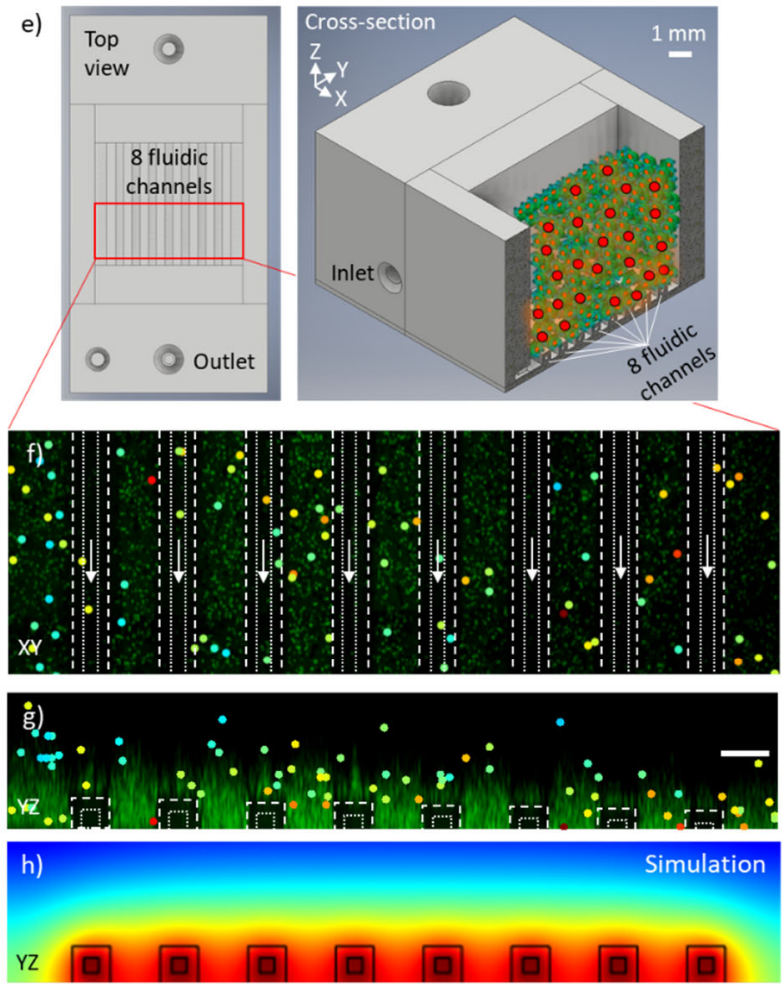

Fig. 6 (a) Oxygenation in 3D tissue model with a single perfused fluidic channel (inner dimensions $140 \times 140$ $\mu \mathrm{m}^{2}$ ) in culture chips manufactured by 3D printing of a hydrogel open to oxygen diffusion. The culture enclosure is seeded with $5 \times 10^{6} \mathrm{Hep} \mathrm{G2}$ cells $/ \mathrm{mL}$ and sensor beads in a GelMA hydrogel. The channel is perfused at an average velocity of $3.2 \mathrm{~mm} / \mathrm{s}$ prior to oxygen mapping. (b) Projected bottom view (XY) of sensor beads at all elevations overlaid on a projected confocal fluorescence micrograph of live stained cells (green). The dotted and dashed lines illustrate the inside and outside, respectively, of the channel walls, and the arrow shows the flow direction. (c) Corresponding cross-sectional views (YZ) with all beads and cell fluorescence projected onto the vertical plane. (d) Cross-section view of a 3D numerical simulation of the predicted oxygen distribution considering both cellular oxygen consumption and oxygen supply by medium perfusion. (e) Oxygenation in a 3D tissue model with an array of 8 perfused microfluidic channels (inner dimensions $140 \mathrm{x}$ $140 \mu \mathrm{m}^{2}$ ). Equal manufacture and culture conditions except for seeding $1 \times 10^{6} \mathrm{Hep} \mathrm{G2} \mathrm{cells/mL} \mathrm{and} \mathrm{a}$ channel perfusion velocity of $3.2 \mathrm{~mm} / \mathrm{s}$. (f) Projected bottom view of sensor beads at all elevations overlaid on a projected confocal fluorescence micrograph of live-stained cells. (g) Corresponding cross-sectional views with all beads and cell fluorescence projected onto the vertical plane. (h) Cross-section of a 3D numerical simulation of the predicted oxygen distribution.

The single channel design is clearly inadequate for supplying oxygen to a 3D cell culture volume at the cell densities used in Fig. 6a-d. We next made use of the numerical simulation results to predict a suitable combination of channel array spacings and seeding cell density to reach physoxic $\left(6 \% \mathrm{O}_{2}\right)$ or higher oxygenation levels in the lowermost $2 \mathrm{~mm}$ of cell culture volume. The resulting COMSOL 
model having 8 channels with a center-to-center pitch of $830 \mu \mathrm{m}$ was transferred into a 3D printable chip design, shown in Fig. 6 e. Printed chips were seeded with $1 \times 10^{6}$ cells $/ \mathrm{mL}$ in GelMA hydrogel, as used in the predictive COMSOL model, and the chips connected to an external peristaltic pump were cultured in the mini-incubator at a channel perfusion rate of $3.2 \mathrm{~mm} / \mathrm{s}$. Fig. $6 \mathrm{f}$ and g show representative lateral and cross-section projections of the measured oxygen distribution overlaid on projected green fluorescence from live staining. The lateral projection suggests significant spatial and apparently inconsistent variation in oxygen tension across the observed volume. However, the cross-sectional projection clearly shows that the variation is only in the vertical direction, monotonically decaying as expected, which would not have been possible to discern using simpler 2D oxygen mapping methods. Comparison of the lateral projection of oxygen readings from sensor beads in Fig. $6 \mathrm{~g}$ and the results of the numerical simulation in Fig. $6 \mathrm{~h}$ used for designing the microchannel array shows excellent agreement between simulation and experiment, with oxygen concentrations consistently above $6 \%$ in the lowermost $2 \mathrm{~mm}$ of the culture volume. Of note, equal cellular oxygen consumption rates in 3D cultures at different cell densities is not a given, ${ }^{34}$ but appears to match the observations in Fig. 6. The optically accessible chip system with cells and sensor beads embedded in 3D may be employed to study such predicted cell density dependent consumption rates in full 3D detail.

\section{Tunable oxygen gradients enabled by 3D oxygen mapping}

Oxygen concentration gradients are present in all solid tissues, with liver sinusoids being a particularly well-studied example. Axial oxygen gradients along capillaries result from the balance between supply through advective transport of oxygenated liquid and consumption by tissue cells in the capillary surroundings. Varying the advective flow rate in 3D printed microperfusion models can dynamically modulate the slope of the steady state concentration gradient. However, the required perfusion rate to maintain a targeted gradient slope may change during culture due to cell proliferation, differentiation, or maturation. Additionally, oxygen tensions will both decrease axially along and radially out from the supply channel in a way that is difficult to predict numerically over time, since both metabolic activity and cell proliferation rates depend on the local oxygen concentration. These problems can be circumvented by the introduction of sensor beads during cell seeding followed by periodic monitoring of the 3D oxygen distribution and subsequent adjustment of the medium perfusion rate to match the targeted 3D gradient(s). Fig. 7 presents results from varying the perfusion rate in the 8-channel microfluidic chip design of Fig. 6e with equal cell seeding and culture perfusion conditions. Static flow conditions cause a nearly anoxic cell environment (Fig. 7b), while very high perfusion rates generate a physoxic to normoxic environment in the entire observed volume, i.e., the lowermost $2.5 \mathrm{~mm}$ of the embedded cell culture, without significant axial variation (Fig. 7e). Intermediate flow rates result in steady state axial and radial gradients outside the flow channels that can be fine-tuned to nearly match the low oxygen base level of brain and thymic tissue $\left(4 \%\right.$ to $2 \% \mathrm{O}_{2}$, Fig. $7 \mathrm{c}$ ) ${ }^{35,36}$ or the higher base level of liver sinusoids ( $6.6 \%$ to $2.6 \%$ in tissue, Fig. 7d), ${ }^{3}$ as physiologically relevant examples. The oxygen concentration distribution was also numerically modelled, and the results are compared to the experimental observation in ESI Fig. S9. 
a)
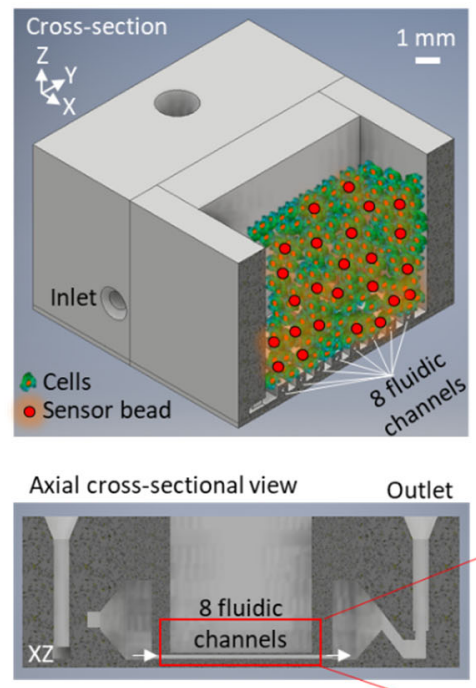
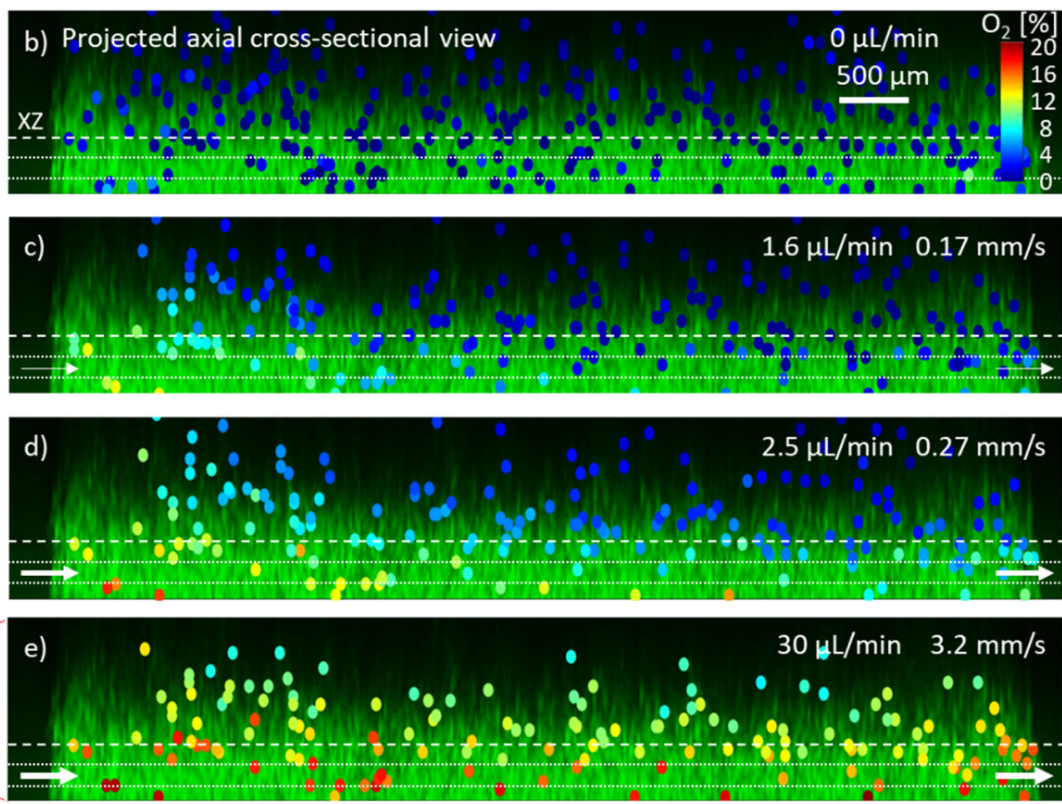

Fig. 7 Defined axial oxygen concentration gradients can be engineered and monitored by varying the channel perfusion rate in (a) a microfluidic 8-channel perfusion system supporting 3D cell culture (identical seeding and culture conditions as in Fig. 6e-g), ranging from (b) anoxic conditions at static conditions over (c and d) gradients mimicking physiological conditions to (e) axially independent oxygenation approaching ambient oxygen levels at very high perfusion rates. The perfusion rates and velocities are for each of the 8 channels.

\section{Oxygen mapping in 4D}

In vitro models of ischemia and ischemia-reperfusion are important to understand cellular processes involved in acute pathological conditions such as myocardial infarction and stroke. ${ }^{37,38}$ Tissue oxygen depletion occurs on sub-minute time scales after complete obstruction of blood flow, calling for a 3D analysis scheme with similar time resolution (4D mapping) in tissue models. The developed 3D oxygen mapping process can match the temporal requirement for sample volumes larger than 1 $\mathrm{mm}^{3}$. Fig. 8 shows selected snapshots of a time series of 3D oxygen mappings (volume map of 1.6 $\mathrm{mm}^{3}$ ), starting with a 3D culture continuously perfused in an 8-channel chip for 14 days with high viability (Fig. 8b, lateral and cross-sectional projections) when perfusion is abruptly stopped. The time-dependent decrease in oxygen tension throughout the culture volume is obvious in subsequent maps collected at a minimum time interval of $47 \mathrm{~s}$ (Fig. 8c-g). Statistical analysis of all measured sensor beads (17-22 automatically detected depending on the local oxygen tension) show initial physoxic culture conditions (mean value of $9 \% \mathrm{O}_{2}$ ) that decrease to form a nearly anoxic environment (mean value of $<2 \% \mathrm{O}_{2}$ ) within $350 \mathrm{~s}$ after terminating perfusion (Fig. 8h). The time scale is similar to that observed for oxygen depletion in human tissue, although it should be noted that the cell density used in this demonstrator model is much lower than real tissue, the channel spacing is much larger than inter-capillary distances, and the oxygen carrying capacity of culture medium is much lower than blood due to the absence of hemoglobin. 
a)

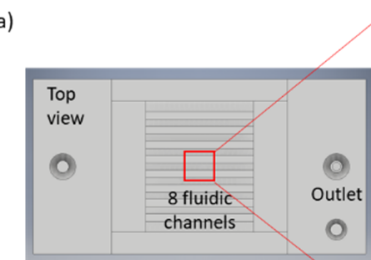

h)

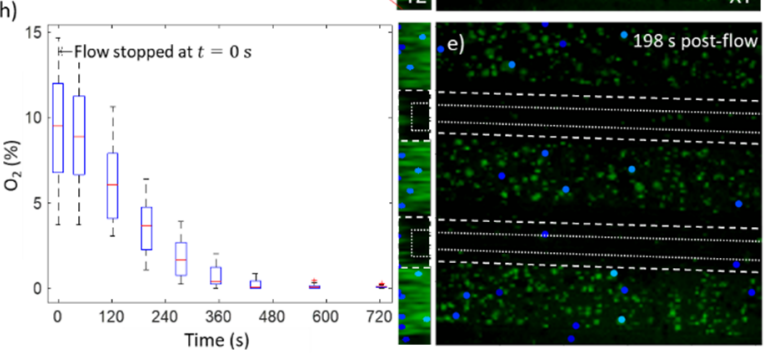

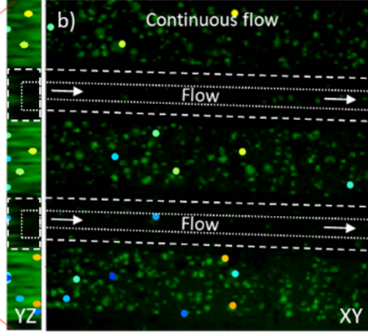
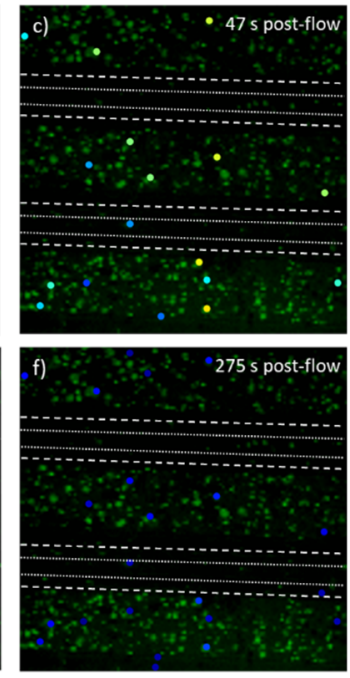
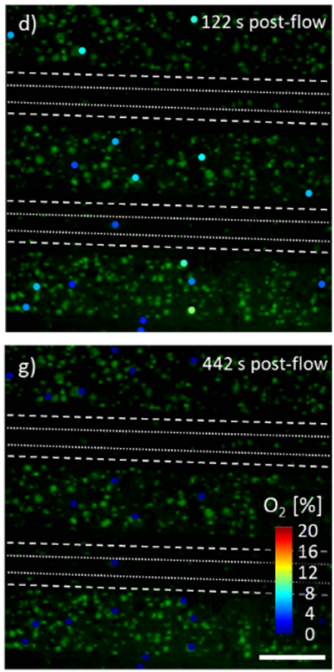

Fig. 8 Oxygen concentration kinetics can be followed on sub-minute time scales, (a) as demonstrated in a microfluidic 8-channel perfusion system with identical seeding and culture conditions as in Fig. 6e-g. (b) The 3D cell culture proceeds with continuous flow for 14 days (cross-sectional and lateral projections of all sensor beads overlaid on green staining for live cells), before stopping the flow and (c-g) monitoring the post-flow oxygen tension development in the surrounding culture volume. (h) Statistical analysis of the time-dependent oxygen tensions obtained through sensor bead readouts show the development of anoxic conditions in $<10$ min post-flow. Red bars show the mean, boxes the 25-75 percentile, and whiskers the full data range at each time point. Scale bar $500 \mu \mathrm{m}$.

\section{Conclusions}

In summary, we have established a robust experimental and analytical procedure to monitor oxygen concentration distributions in 3D cell cultures at high cell densities and to depths above $1 \mathrm{~mm}$. The procedure can be applied in week-long cultures without indications of cytotoxicity, and can track volumetric changes occurring on sub-minute time scale. The method can additionally be used as a microfluidic design tool to assess, model, and predict the actual oxygen concentration distribution for a specific choice of cultured cells, cell densities, and cell embedding media, as demonstrated for the single or multi-channel array design. Lastly, the ability to perform non-invasive oxygen mapping with true 3D resolution allows for the generation and dynamic maintenance of oxygen concentration gradients in 3D tissue models throughout the culture period.

\section{Materials and Methods}

\section{Oxygen sensor beads}

Oxygen sensor beads CPOx-50-PtP (CPOx Red, oxygen-sensing platinum-porphin complex and singlet oxygen scavengers embedded in polystyrene) were purchased from Colibri Photonics (Colibri Photonics $\mathrm{GmbH}$, Potsdam, Germany). The sensor beads were pre-coated and sterilized before use in tissue cultures. Briefly, $10 \mathrm{mg}$ of the sensor beads were immersed in $2 \mathrm{ml}$ of $1 \%$ BSA (A4161, Sigma Aldrich) in DPBS (D8537, Sigma Aldrich) solution, vortexed and incubated for $1 \mathrm{~h}$. Then, sensor beads were sterilized by immersion in $70 \%$ ethanol and incubated for $1 \mathrm{~h}$, vortexed 3-4 times during incubation. After BSA pre-coating and sterilization with ethanol the sensor beads were washed 3-4 times with DPBS to remove residual ethanol by centrifugation, removal of supernatant and reimmersion in $2 \mathrm{~mL}$ DPBS. Finally, the sensor beads were dispersed in DPBS at a concentration of 5 $\mathrm{mg} / \mathrm{mL}$.

\section{Design and 3D printing of the organ model chips}


3D hydrogel chips were designed using CAD software (Inventor Professional 2019, Autodesk, USA). The 3D stereolithographic hydrogel printing method, cytotoxicity assessment and perfusion setup have been described in our previous work. ${ }^{4}$ The pre-polymer solution was adapted and consisted of $200 \mathrm{mg} / \mathrm{mL}$ poly(ethylene glycol) diacrylate (PEGDA; $\mathrm{M}_{\mathrm{n}} 700 \mathrm{~g} / \mathrm{mol}$; Sigma-Aldrich), $11.25 \mathrm{mg} / \mathrm{mL}$ quinoline yellow (Sigma-Aldrich), $5 \mathrm{mg} / \mathrm{mL}$ lithium phenyl-2,4,6-trimethylbenzoylphosphinate (LAP, Sigma-Aldrich) in water and optimized exposure settings of $4.5 \mathrm{~s}$ per printed layer with a step height of $20 \mu \mathrm{m}$.

\section{Maintenance of cell line}

Hep G2 human hepatocyte carcinoma cells (ECACC Cat. No. 85011430) were cultured in complete medium consisting of EMEM (EBSS) supplemented with $2 \mathrm{mM}$ glutamine (L-glutamine solution, CAS No. 56-85-9, BioXtra; G7513, Sigma-Aldrich), 1\% non-essential amino acids (MEM Non-essential amino acid solution, BioReagent; M7145 Sigma-Aldrich), 10\% v/v FBS (S1810, Biowest, France) and $1 \% \mathrm{v} / \mathrm{v}$ penicillin-streptomycin (Sigma-Aldrich). Cells were cultured in T75 and T175 culture flasks at $5 \% \mathrm{CO}_{2}$ and $37{ }^{\circ} \mathrm{C}$ and medium was exchanged every $3-4$ days. Cell were passaged at $80 \%$ confluency and used between passage 5 and 20 for all presented experiments.

\section{D adhesion culture in 96-well plate}

Hep G2 were seeded at $30 \times 10^{3}$ cells/well in $200 \mu \mathrm{L}$ of complete medium in Nunclon-Delta treated 96-well plate (167008, Thermo Fisher Scientific). Viability (live) assays were performed using $1 \mu \mathrm{g} / \mathrm{mL}$ calcein-AM (65-0853, Invitrogen) in FBS-free medium for $1 \mathrm{~h}$. Following live staining, $50 \mu \mathrm{L}, 100 \mu \mathrm{L}$ and $200 \mu \mathrm{L}$ of complete medium were added to the wells of each treatment group, and an additional $2 \mu \mathrm{L}$ of $5 \mathrm{mg} / \mathrm{mL}$ CPOx Red oxygen sensor beads were added per well for oxygen mapping.

\section{D hemispheres in 96-well plate}

The cell suspension contained $20 \times 10^{6} \mathrm{Hep} \mathrm{G2} / \mathrm{ml}$ in $7.5 \%$ GelMA (GelMA Lyophilizate, Cellink), 0.5 $\mathrm{mg} / \mathrm{mL}$ CPOx Red oxygen sensor beads, $1 \mathrm{mg} / \mathrm{mL}$ LAP in complete medium. A droplet of $11 \mu \mathrm{L}$ was pipetted onto the bottom of each well in a 96-well plate and cured from the bottom side with a custom-built UV (365 nm, $\left.21 \mathrm{~mW} / \mathrm{cm}^{2}\right)$ light source for $1 \mathrm{~min}$, initiated no later than $30 \mathrm{~s}$ after droplet deposition to avoid sedimentation of cells and sensor beads. After UV curing, $150 \mu \mathrm{L}$ of complete medium was added to the first set of samples. To the second set of samples, a solution of $40 \mu \mathrm{L}$ of $7.5 \%$ GelMA, $0.5 \mathrm{mg} / \mathrm{mL}$ CPOx oxygen sensor probes, $1 \mathrm{mg} / \mathrm{mL}$ LAP in complete medium was added and cured for 1 min using the custom-built UV light source. After curing, $110 \mu \mathrm{L}$ of complete medium was added.

\section{D perfusion chips}

Hep G2 were seeded at a concentration of $1.0 \times 10^{6}$ cells $/ \mathrm{mL}$ and $0.2 \times 10^{6}$ in $7.5 \%$ GelMA, 0.5 $\mathrm{mg} / \mathrm{mL}$ CPOx Red oxygen sensor beads, $1 \mathrm{mg} / \mathrm{mL}$ LAP in complete medium into voids of 3D printed, perfusable cell culture scaffolds. A volume of $100 \mu \mathrm{L}$ was seeded per well of 3D printed hydrogel chips and cured with the custom-built UV light source for $1 \mathrm{~min}$ from bottom and top, initiated no later than $30 \mathrm{~s}$ after seeding to avoid sedimentation of cells and oxygen sensor beads. The cell culture chips were cultured fully immersed in $9 \mathrm{~mL}$ of complete medium in a 6-well plate, and medium was exchanged every 3 days. For co-localization of live cells and oxygen sensor beads, cells were stained by perfusion of $2 \mu \mathrm{g} / \mathrm{mL}$ calcein-AM in complete medium for $3 \mathrm{~h}$. For chip perfusion, tubing with inner diameter $0.51 \mathrm{~mm}$ (GRA PPR0051008, Gradko) in combination with pump stopper tubing (SC0305A, Cole-Parmer) and peristaltic pump (LabV1, Shenchen) was used.

\section{Phosphorescence lifetime microscopy measurements}


Image stacks of confocal fluorescence and phosphorescence lifetime measurements were acquired with a DCS-120 Confocal FLIM System (Becker \& Hickl, Germany) connected to an Axio Observer Z1 (Zeiss, Germany) with a Fluar 5x/0.25NA (Carl Zeiss) objective. Two parallel photon detectors were used to decrease the incident photon flux per detector and thus the effect of detector dead-time. Tile images were recorded using a $\mathrm{H} 117$ motorized stage (Prior, England). Image processing scripts were written in and performed with MATLAB (Mathworks, USA). Microscope stage incubators were manufactured in-house and allowed for stable culture conditions during microscopy, namely a temperature of $36 \pm 1{ }^{\circ} \mathrm{C}$ and ambient gas mixture of $95 \%$ air $/ 5 \% \mathrm{CO}_{2}$, premixed using mass flow controllers (type 1179A, MKS).

\section{Data processing}

The image files containing fluorescence and phosphorescence lifetime images were directly imported to MATLAB with a custom-written script. Voxels with less than 5 photons in the phosphorescent part of their decay trace (182 time bins; $220 \mu \mathrm{s}$ ) were excluded from further analysis. Voxels with connecting faces were grouped to regroup the signal from beads. To separate beads in close proximity, voxels with intensities below $20 \%$ of the maximum intensity voxel in each bead were deleted and the bead grouping algorithm was re-applied. Then, decay traces from each bead were summed. Summed decay traces were corrected for detector saturation and the highest intensity plane and horizontal center point of grouped objects was determined for bead positioning in 3D oxygen maps.

\section{Data Analysis}

Decay traces were fitted using maximum likelihood estimation with single, biexponential and stretched exponential functions with background. The information from calibration curves was used to correlate lifetimes with oxygen tensions for each bead. 3D oxygen maps were plotted with color coded oxygen tensions. Oxygen maps are visualized stand-alone or with co-localized live-stained 3D intensity and optionally separately processed fluorescence lifetime images.

All scripts for the presented oxygen mapping technique were written in MATLAB (Mathworks, USA).

\section{Conflicts of interest}

There are no conflicts to declare.

\section{Acknowledgements}

Financial support from Independent Research Fund Denmark, grant 7017-00366B and from Technical University of Denmark, Department of Health Technology is kindly acknowledged. 


\section{References}

1 T. L. Place, F. E. Domann and A. J. Case, Free Radic. Biol. Med., 2017, 113, 311-322.

2 A. Al-Ani, D. Toms, D. Kondro, J. Thundathil, Y. Yu and M. Ungrin, PLoS One, 2018, 13, 1-13.

3 T. Kietzmann, Redox Biol., 2017, 11, 622-630.

$4 \quad$ R. Zhang and N. B. Larsen, Lab Chip, 2017, 17, 4273-4282.

$5 \quad$ X. Wang and O. S. Wolfbeis, Chem. Soc. Rev., 2014, 43, 3666-3761.

$6 \quad$ M. A. Yaseen, V. J. Srinivasan, S. Sakadžić, W. Wu, S. Ruvinskaya, S. A. Vinogradov and D. A. Boas, Opt. Express, 2009, 17, 22341.

7 K. Mizukami, A. Katano, S. Shiozaki, T. Yoshihara, N. Goda and S. Tobita, Sci. Rep., 2020, 10, 114.

8 A. V. Kondrashina, R. I. Dmitriev, S. M. Borisov, I. Klimant, I. O’Brien, Y. M. Nolan, A. V. Zhdanov and D. B. Papkovsky, Adv. Funct. Mater., 2012, 22, 4931-4939.

9 A. Raza, H. E. Colley, E. Baggaley, I. V. Sazanovich, N. H. Green, J. A. Weinstein, S. W. Botchway, S. MacNeil and J. W. Haycock, Sci. Rep., 2017, 7, 1-9.

P. Lehner, C. Staudinger, S. M. Borisov, J. Regensburger and I. Klimant, Chem. - A Eur. J., 2014, 21, 3978-3986.

11 M. A. Acosta, P. Ymele-Leki, Y. V. Kostov and J. B. Leach, Biomaterials, 2009, 30, 3068-3074.

12 L. Wang, M. A. Acosta, J. B. Leach and R. L. Carrier, Lab Chip, 2013, 13, 1586-1592.

13 S. C. Lesher-Pérez, G. A. Kim, C. H. Kuo, B. M. Leung, S. Mong, T. Kojima, C. Moraes, M. D. Thouless, G. D. Luker and S. Takayama, Biomater. Sci., 2017, 5, 2106-2113.

14 E. Schmälzlin, J. T. van Dongen, I. Klimant, B. Marmodée, M. Steup, J. Fisahn, P. Geigenberger and H.-G. Löhmannsröben, Biophys. J., 2005, 89, 1339-1345.

15 S. Prill, D. Bavli, G. Levy, E. Ezra, E. Schmälzlin, M. S. Jaeger, M. Schwarz, C. Duschl, M. Cohen and Y. Nahmias, Arch. Toxicol., 2016, 90, 1181-1191.

16 H. Xu, J. W. Aylott, R. Kopelman, T. J. Miller and M. A. Philbert, Anal. Chem., 2001, 73, 41244133.

17 C. Gehre, M. Flechner, S. Kammerer, J. H. Küpper, C. D. Coleman, G. P. Püschel, K. Uhlig and C. Duschl, Sci. Rep., 2020, 10, 1-12.

18 R. L. Wilson, J. P. Connell and K. J. Grande-Allen, ACS Biomater. Sci. Eng., 2019, 5, 4522-4530.

19 W. Becker, A. Bergmann, M. A. Hink, K. König, K. Benndorf and C. Biskup, Microsc. Res. Tech., 2004, 63, 58-66.

20 W. Becker, B. Su, A. Bergmann, K. Weisshart and O. Holub, Multiphot. Microsc. Biomed. Sci. $X I, 2011,7903,790320$.

21 E. Schmälzlin, L. Friedmann, E. Horn and R. Zantl, Laser+ Photonics, 2015, 70-73.

22 J. W. Nichol, S. T. Koshy, H. Bae, C. M. Hwang, S. Yamanlar and A. Khademhosseini, Biomaterials, 2010, 31, 5536-5544.

23 J. R. Lakowicz, in Principles of Fluorescence Spectroscopy, Springer US, Boston, MA, 3rd edn., 2006, pp. 97-155.

24 E. R. Carraway, J. N. Demas, B. A. DeGraff and J. R. Bacon, Anal. Chem., 1991, 63, 337-342.

25 Y. Cai, A. Smith, J. Shinar and R. Shinar, Sensors Actuators, B Chem., 2010, 146, 14-22. 
26 P. Hall and B. Selinger, J. Phys. Chem., 1981, 85, 2941-2946.

27 Ž. Bajzer, T. M. Therneau, J. C. Sharp and F. G. Prendergast, Eur. Biophys. J., 1991, 20, 247262.

28 J. Tellinghuisen and C. W. Wilkerson, Anal. Chem., 1993, 65, 1240-1246.

29 H. Akaike, IEEE Trans. Automat. Contr., 1974, 19, 716-723.

30 J. Keizer, J. Am. Chem. Soc., 1983, 105, 1494-1498.

31 R. K. Christensen, C. Von Halling Laier, A. Kiziltay, S. Wilson and N. B. Larsen, Biomacromolecules, 2020, 21, 356-365.

32 L. Kamalian, A. E. Chadwick, M. Bayliss, N. S. French, M. Monshouwer, J. Snoeys and B. K. Park, Toxicol. Vitr., 2015, 29, 732-740.

33 M. Decleer, J. Jovanovic, A. Vakula, B. Udovicki, R. S. E. K. Agoua, A. Madder, S. De Saeger and A. Rajkovic, Toxins (Basel)., , DOI:10.3390/toxins10070266.

34 C. Magliaro, G. Mattei, F. lacoangeli, A. Corti, V. Piemonte and A. Ahluwalia, Front. Bioeng. Biotechnol., 2019, 7, 1-9.

35 J. Dings, J. Meixensberger, A. Jäger and K. Roosen, Neurosurgery, 1998, 43, 1082-1095.

36 L. P. Hale, R. D. Braun, W. M. Gwinn, P. K. Greer and M. W. Dewhirst, Am. J. Physiol. - Hear. Circ. Physiol., 2002, 282, H1467-H1477.

37 G. Khanal, K. Chung, X. Solis-Wever, B. Johnson and D. Pappas, Analyst, 2011, 136, 35193526.

38 H. Liu, O. A. Bolonduro, N. Hu, J. Ju, A. A. Rao, B. M. Duffy, Z. Huang, L. D. Black and B. P. Timko, Nano Lett., 2020, 20, 2585-2593. 


\section{Electronic Supplementary Information}

for

\section{Optical 4D oxygen mapping of microperfused tissue models with tunable in-vivo like 3D oxygen microenvironments}

Milan Finn Wesseler, Mathias Nørbæk Johansen, Aysel Kızıltay, Kim I. Mortensen, and Niels B. Larsen*

Department of Health Technology, DTU Health Tech, Technical University of Denmark, 2800 Kgs. Lyngby, Denmark

E-mail: nibl@dtu.dk 


\section{Vertical accuracy of the bead position analysis}

The lateral position of each sensor bead can be determined with high accuracy, while the accuracy of its vertical position is limited by the numerical aperture of the objective and by the optical properties of the bead material. The apparent vertical extent can be assessed from analysis of a confocal stack of sensor beads embedded in a hydrogel at different heights. The presence of embedded cells might influence the optical quality, so the analysis was performed on a hemispherical droplet as also analyzed in the main text (Fig. 2). Fig. S1a shows overlaid fluorescence and phosphorescence, while Fig. S1b only shows the phosphorescence. The dashed line indicates the location for performing a vertical cross-section through a random bead shown in the insert below (labeled XZ). Fig. S1c is a zoom on the cross-sectional view of that bead, suggesting an apparent vertical extent of 200-300 $\mu \mathrm{m}$ and with a pronounced intensive maximum spanning approximately $100 \mu \mathrm{m}$.

Acquisition of each confocal stack uses a physical height difference of $20.0 \mu \mathrm{m}$. This corresponding optical path length difference between imaging planes is taken to be $26.6 \mu \mathrm{m}$, approximating the refractive index of the hydrogel surroundings by that of pure water $(n=1.33)$. The apparent vertical extent of 6 random beads is investigated quantitatively, with the selection criterion being that they have their maximum observed phosphorescence intensity at an image plane separated by 5 imaging planes from another bead. Fig. S1d presents the results of the analysis. The horizontal axis shows the depth (image plane) where each of the beads has the largest summed photon count, i.e., the bead center. The vertical axis shows the sampling depth range (image planes) for all 6 beads. Each horizontal bar in the violin plot represents the relative summed photon count in that image plane. The quantitative analysis suggests an extent of the intensity maximum of no more than 2 image planes, i.e., approximately $45 \mu \mathrm{m}$ in vertical extent, and it is obvious that fitting of the summed counts from all vertical position allows for highly accurate prediction of the vertical intensity maximum.

Accurate placement of color-coded oxygen sensor depictions in 3D volumes was evaluated by overlaying $\mathrm{XY}$ and $\mathrm{XZ}$ phosphorescence maximum intensity projection images with oxygen sensor maps, shown in Fig. S1e+f. Both $X Y$ and $Z$ oxygen sensor depictions are observed to be co-located with corresponding phosphorescent signal from the original oxygen sensor beads in the sample volume. 


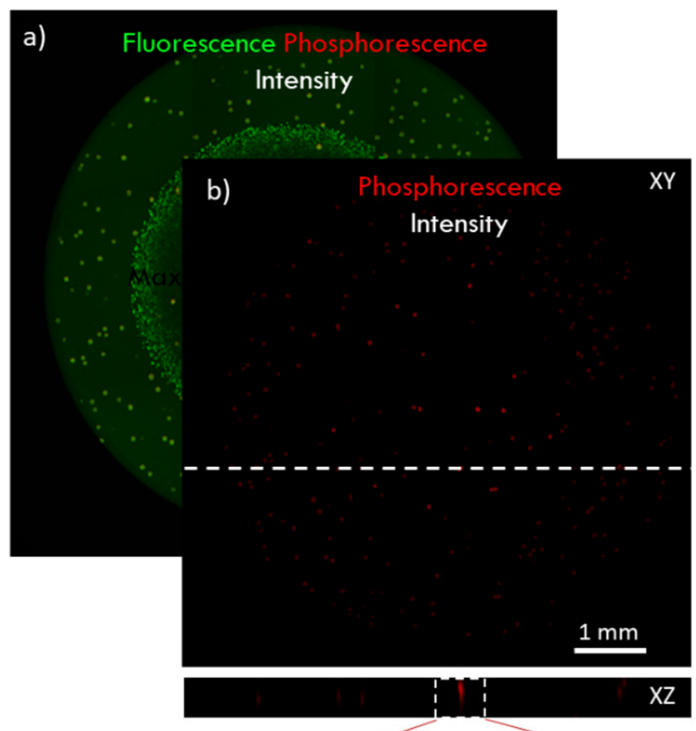

d)

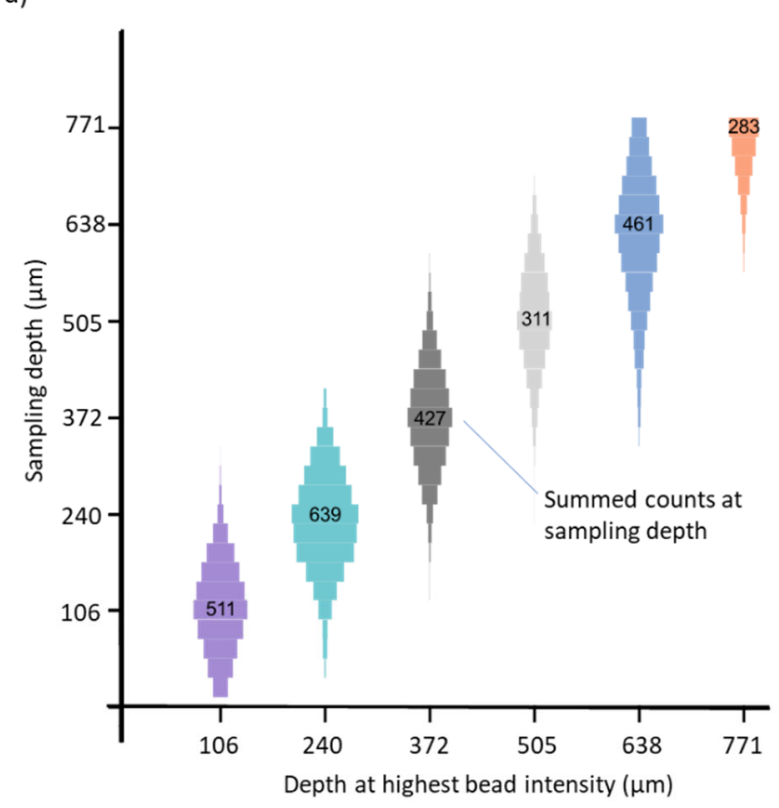

$200 \mu \mathrm{m}$

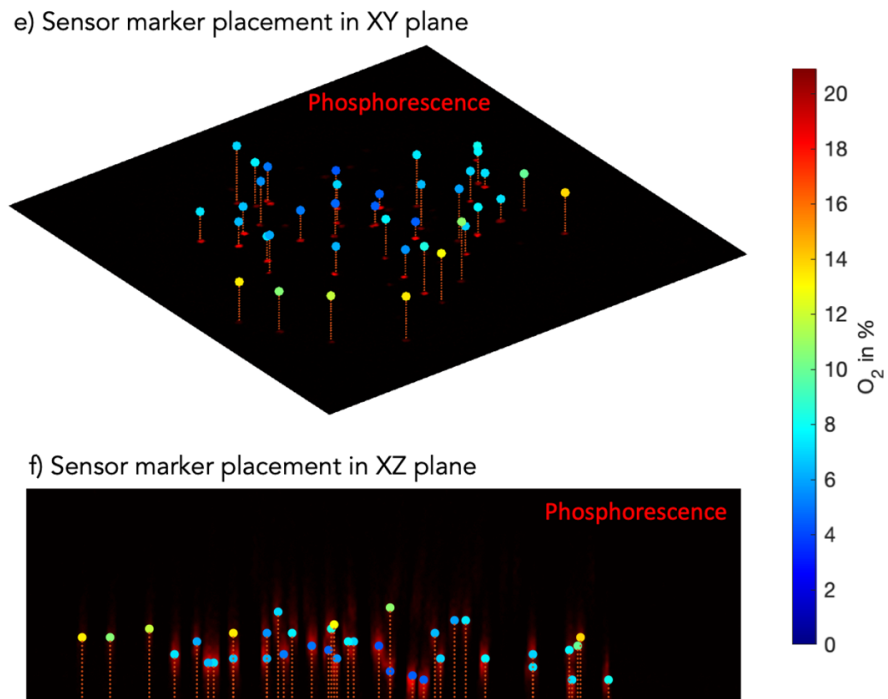

Fig. S1 Analysis of the apparent vertical extent of sensor beads. (a) Overlaid maximum projections of fluorescence and phosphorescence from a hemispherical GeIMA hydrogel with embedded sensors beads (green and red) and live-stained cells (green). (b) A single image plane (XY) of the underlying confocal stack showing only phosphorescence from the sensor beads. The dashed line indicates where the cross-sectional plane at the bottom (XZ) is extracted. (c) Five times magnification of the outlined part of the XZ cross-section, showing the vertical and lateral extent of a single sensor bead as observed in the confocal stack. (d) Vertical intensity distribution (summed photon counts) of 6 beads having their highest photon counts at different depths (horizontal axis). The width of each level of the violin plot of each bead shows its relative summed photon counts for varying imaging depths (shown on the vertical axis), with the summed photon count at the plane of its highest photon count specified. $(e, f)$ Maximum intensity projections of 3D phosphorescence intensity image with $256 \times 256 \times 50$ voxels ( 50 z-planes recorded with $20 \mu \mathrm{m}$ stepping) along with oxygen sensor placement. Location on XY plane indicated with red dots below color-coded oxygen sensor depictions. 


\title{
2 Development of the data processing methodology
}

\subsection{Data processing flow}

Signal processing steps: From 4D arrays of photon counts to 3D oxygen maps

\author{
Disregard noise \\ Group phosphorescent signal from each oxygen sensor probe \\ Correct for detector saturation \\ Fit phosphorescent decay curves \\ Correlate to oxygen concentration \\ Plot 3D Oxygen Maps with co-localized live stained cells
}

Fig. S2 Signal processing steps to go from 4D arrays of photon counts to 3D visualizations of oxygen sensor probes.

Fig. S2 shows the main signal processing steps needed to go from 4D arrays containing phosphorescent photon counts to 3D visualizations of oxygen sensor probes. First, the 4D arrays of phosphorescent photon counts were summed in their time dimension and all voxels of the resulting 3D matrix with less than 5 photons were disregarded as background noise. This was above the background signal threshold from the microscope without samples and typically decreased the fraction of remaining voxels to $<1 \%$. Next, 3D photon intensity maps were transformed into a 3D binary image ( 1 for $>0$ photons, 0 otherwise) and objects that consisted of more than 15 voxels with connected faces were identified. Given the size of the oxygen sensor beads $(\varnothing 50 \mu \mathrm{m})$, objects with less than 15 voxels were considered out of focus beads or background. Some beads in close proximity were grouped into a single object when applying the low noise threshold of 5 photons only. To separate incorrectly grouped beads, voxels in each grouped object with less than $20 \%$ of the maximum intensity voxel contained in that same object were deleted. Afterwards, the grouping algorithm was re-applied, again creating objects with $>15$ voxels with connected faces that usually consisted of single beads then. Since single voxels in each object (or each oxygen sensor bead) contained low numbers of photons in their decay traces (typically 10-150 photons), decay traces from all voxels in each bead were grouped to retrieve one decay trace per bead. Beads with less than 1000 photons total were deleted to ensure sufficient photon number for data fitting with low errors. The corresponding analysis is presented below.

Since each detection unit of the single photon counting system had a specified dead-time of $100 \mathrm{~ns}$ after each registered photon event, the beginning of decay curves where intensities were still higher typically suffered from photon loss which skewed the consecutive fit and lead to a decrease in fitted lifetime with increasing intensity. Thus, the decay traces were corrected for detector saturation. The lowest change in oxygen tensions when recording samples with 10 -fold increases in intensity were observed when using a dead time of $t_{d}=55 \mathrm{~ns}$. The system contained 2 parallel detection units with a polarization splitter in the optical.

Then, decay traces for each bead were fitted and the retrieved lifetime parameter was translated into an oxygen tension through previously recorded calibration curves. The oxygen sensor beads 
were then plotted in 3D at the center of the XY position of the 3D object and the Z-plane with the highest photon intensity. Live cell maps acquired in parallel were overlayed.

\subsection{Time-dependent data from multiple voxels within a sensor bead can be summed}

The acquired data consists of photon counts distributed in discrete time "bins" ( 256 in the setup used) after excitation of each voxel. As discussed in the main text, the number of photons arriving from a single voxel after a single excitation cycle is insufficient for high-quality curve fitting. Thus, the binned photon counts of multiple voxels within each bead should be summed by bin to provide better total counting statistics. This is only possible if all voxels of each bead do indeed have equivalent intensity decay profiles. We examined the degree of equivalence extensively within the spatial extent of each bead for multiple beads and for multiple dissolved oxygen concentrations. Formally, this is an assessment of whether the time-dependent photon count distribution from all bead voxels is drawn from the same Poisson distribution. Fig. S3 shows representative examples of the observed intensity profiles (blue bars) from 4 distinct voxels within one bead at dissolved oxygen concentrations of $21 \%$ (a) or $0 \%$ (b), each intensity distribution resulting from the summation of 20 repeated acquisitions at each voxel. The summed intensity distribution from each voxel was subsequently fitted by the decay profile found to be optimal (bi-exponential intensity decay with a predetermined background, see ESI section 2.4). The parameters of the fitted profile to each voxel's intensity decay curve are found to be statistically indistinguishable from the profile acquired at the other three voxel locations, at either oxygen concentration investigated. This supports the assumption of the time-dependent intensity distribution of different voxels within a bead being drawn from the same Poisson distribution, and that bin-wise summation of the photon count distributions of separate voxels is analytically valid. 
a)
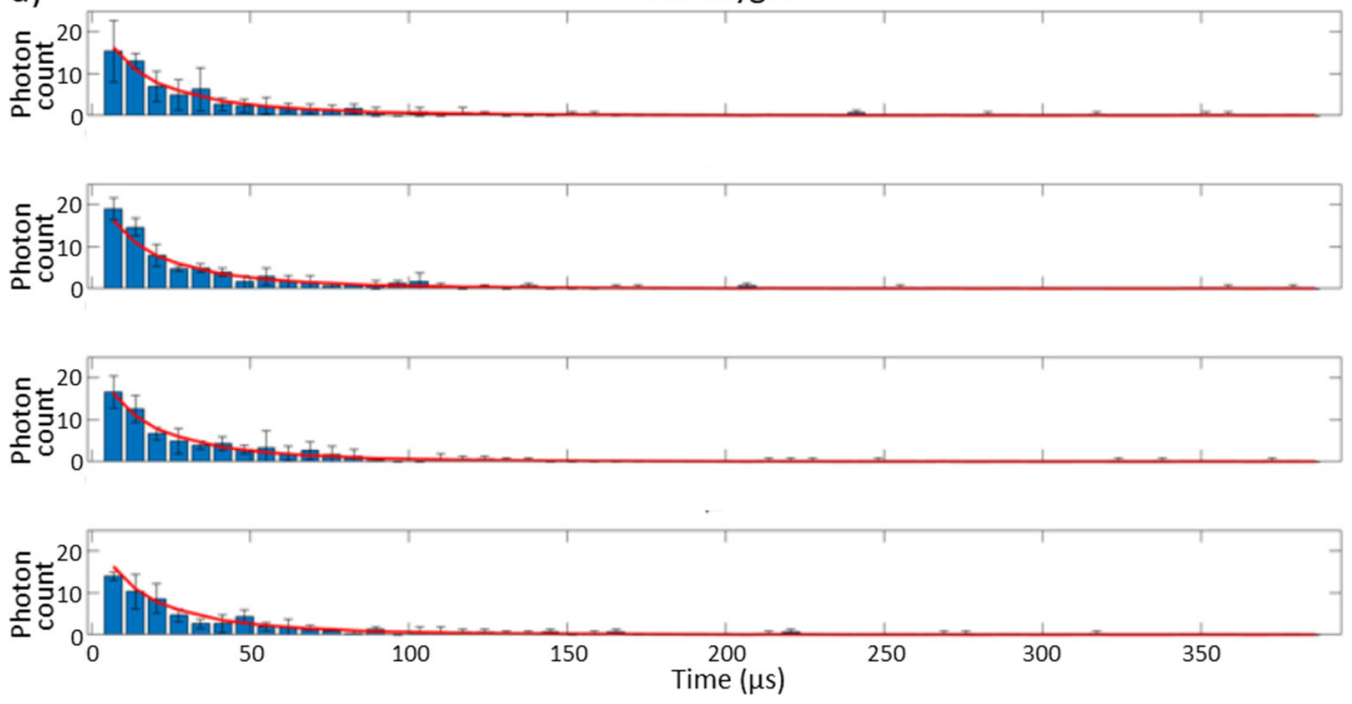

b)

$0 \%$ oxygen
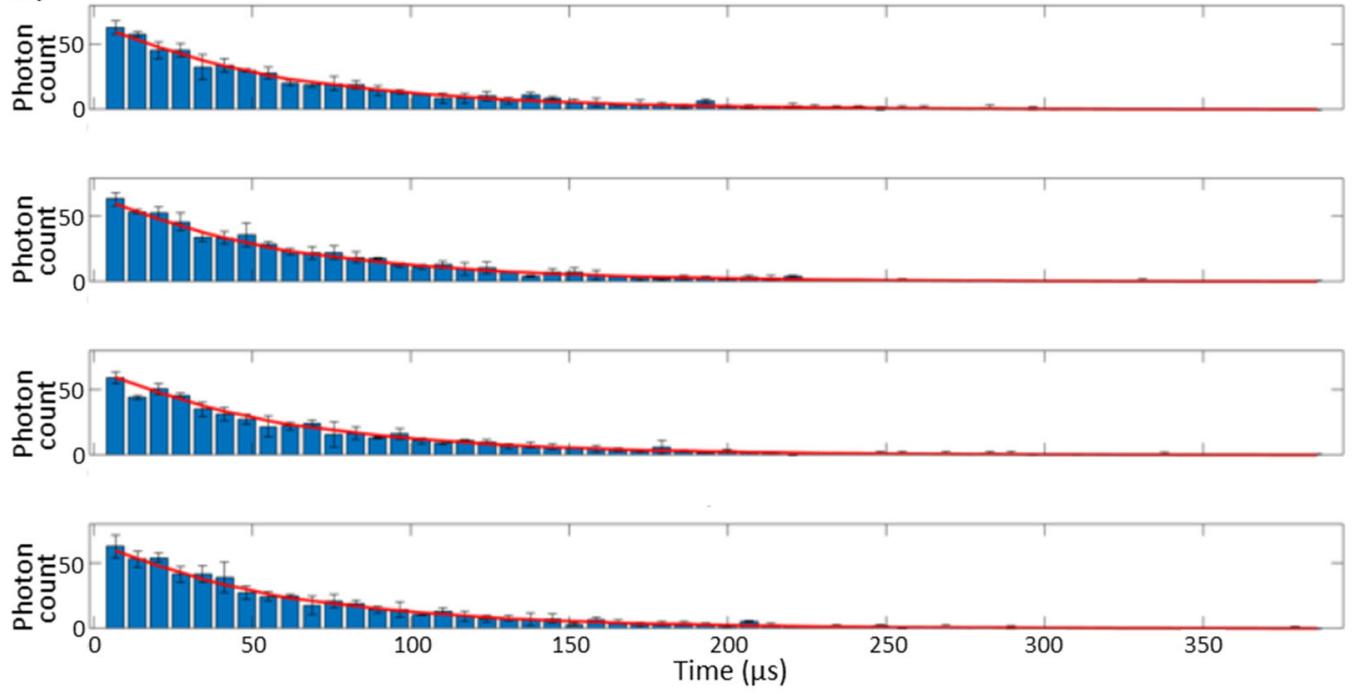

Fig. S3 The four graphs for each oxygen concentration show the recorded data (blue bars) with standard deviation error bars of the mean, and with the mean (red line) across all pixels belonging to that bead. The 4 randomly chosen pixels that are shown for each oxygen concentration illustrate that the data from each individual pixel seems to originate from the same distribution. The plots shown in this Fig. represents what was a general observation on multiple datasets. The data set was recorded by summing 20 image frames at the same imaging height. 


\subsection{Non-linear least squares fitting cannot capture the intensity distribution at low photon counts}

Intensity decay profiles have most commonly been fitted using non-linear least squares fitting (NLLS) protocols that assume a normal distribution of data values. This is a good approximation for high photon counts, using extended acquisition times, where the underlying Poisson distribution becomes indistinguishable from the normal distribution. However, the approximation fails at the low photon counts resulting from the targeted fast mapping of a 3D volume. Fig. S4 compares three commonly used NLLS fitting functions for intensity decay, (i) a single exponential (red curve), (ii) a stretched exponential (green curve), and (iii) a biexponential with a predetermined background (purple curve), applied to data recorded on a sensor bead at $21 \%$ oxygen. The photon counts are presented on a logarithmic scale to highlight the low-intensity observations at longer decay times. The single exponential poorly fits the observations throughout the decay time. Both the stretched exponential and the biexponential fitted by NLLS follow the observations at high photon counts but fail to correctly describe the tail of the decay profile. In contrast, Maximum Likelihood Estimation (MLE) works for any type of statistical distribution including the Poisson distribution and with NLLS being the limiting case of MLE of a Poisson distribution for large numbers of observations. This is illustrated in Fig. S4, where MLE fitting of a bi-exponential with a predetermined background (see ESI section 2.4) captures the photon count distribution both in the beginning (high photon counts) and the end (low photon counts approaching the background level) of the recorded decay trace. Thus, MLE is the preferred method of fitting the observations and extracting effective lifetimes in the developed analysis protocol.

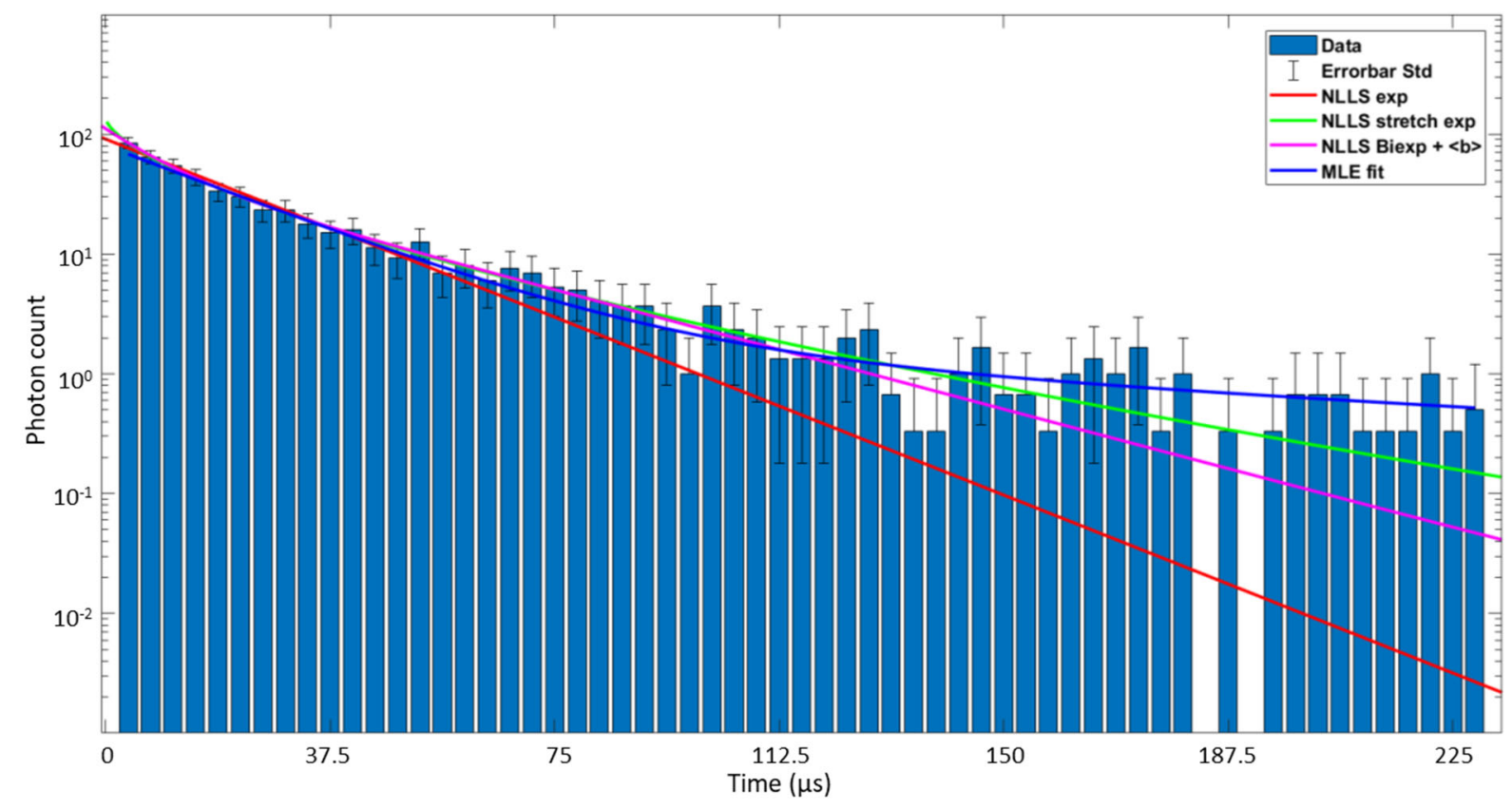

Fig. S4. Comparison of the most used NLLS-based fitting functions in single-photon counting applications and the best performing MLE function. The data originates from a sensor bead at $21 \%$ oxygen. The data has been re-binned from 182 to 61 bins for visualization. Each bar represents the mean of the original bins, and the error bars show the standard deviation of the mean. Comparing the NLLS and MLE bi-exponential fits show the impact of the distribution assumption being normal and Poisson, respectively. 


\subsection{Selection of fitting function by application of the AIC criterion and a derived scoring system}

The Akaike information criterion (AIC) assesses the quality of a fit penalized by the number of free parameters in the fit. Calculated AIC values are smaller for a better fit and/or a smaller number of parameters, which is preferred. We calculated AIC values for each type of fitting function applied to acquired data for each bead at different oxygen concentrations to find the preferred function. The three tested functions are:

- Single exponential: $\left\langle n_{i}\right\rangle=\frac{\langle N\rangle}{\tau} \int_{(i-1) \Delta t}^{\Delta t} \exp (-t / \tau) d t, \quad i=1, \ldots, N$

- Stretched exponential: $\left\langle n_{i}\right\rangle=\frac{\langle N\rangle \beta}{\tau \Gamma\left(\frac{1}{\beta}\right)} \int_{(i-1) \Delta t}^{\Delta t} \exp \left(-[t / \tau]^{\beta}\right) d t, \quad i=1, \ldots, N$

- Bi exponential: $\left\langle n_{i}\right\rangle=\frac{\langle N\rangle}{a \tau_{1}+(1-a) \tau_{2}} \int_{(i-1) \Delta t}^{\Delta t}\left[a \exp \left(-t / \tau_{1}\right)+(1-a) \exp \left(-t / \tau_{2}\right)\right] d t, \quad i=1, \ldots, N$
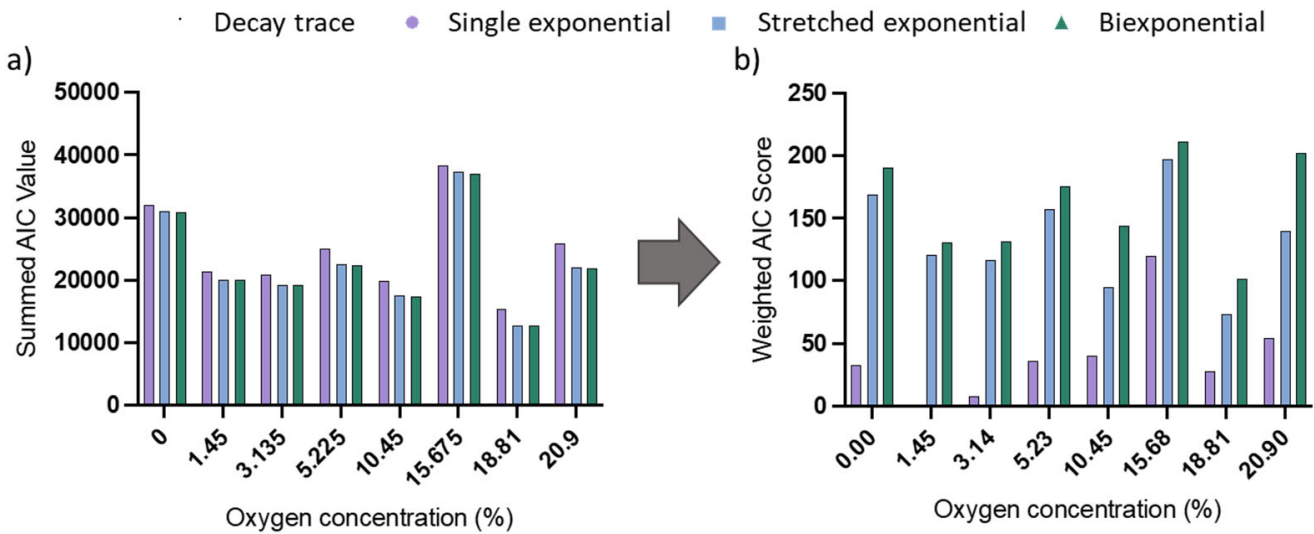

Fig. S5. Selection of fitting function by application AIC criterion and translation into AIC scoring system. (a) Summed AIC values of all beads in measurements at $0 \%$ to $20.9 \%$ oxygen for single, bi- and stretched exponential function and (b) corresponding weighted AIC score.

Fig. S5 shows the summed AIC values for calibration measurements from $0 \%$ to $21 \% \mathrm{O}_{2}$ for single exponential, bi-exponential and stretched exponential functions (Fig. S5a) and the corresponding weighted AIC score (Fig. S5b). As is apparent, the single exponential function had the highest summed AIC value (poorest fit), the stretched exponential the second highest and the bi-exponential function the lowest AIC value (best fit) across all oxygen tensions. The summed AIC value for each fitting function and oxygen tension is retrieved by summing the AIC values of all beads in a measurement. Strong outliers in AIC values from single beads in the bead population of a measurement and fitting function would not be apparent here and could distort the result. Thus, a weighted AIC score was calculated. The scoring system worked as follows: For each single oxygen sensor bead, a single, bi- and stretched exponential fit was applied and the AIC scores were compared. The function with the lowest resulting AIC each individual bead was scored with 3 points, the function with second lowest AIC value got scored with 2 points, and the one with the highest AIC value got 1 point. This way, the fitting function with low AIC values for most oxygen sensor beads could be identified. Fig. S5b confirms that the biexponential function not only has the lowest summed AIC values but also has the highest weighted AIC score across all oxygen tensions, followed by the stretched exponential and with significant gap the single exponential. 


\subsection{Influence of lower intensity cut-off on oxygen measurement accuracy}

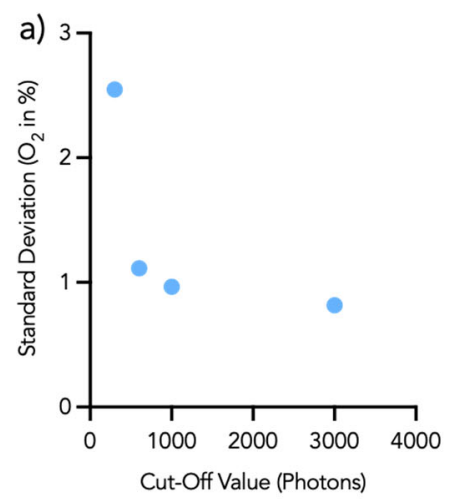

b)
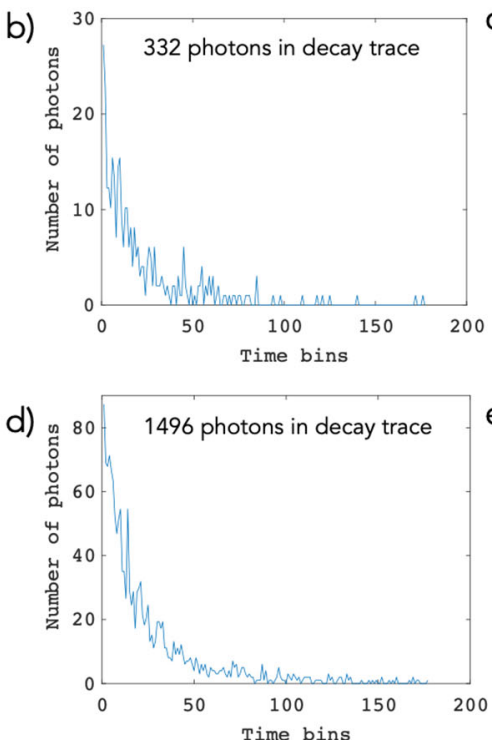
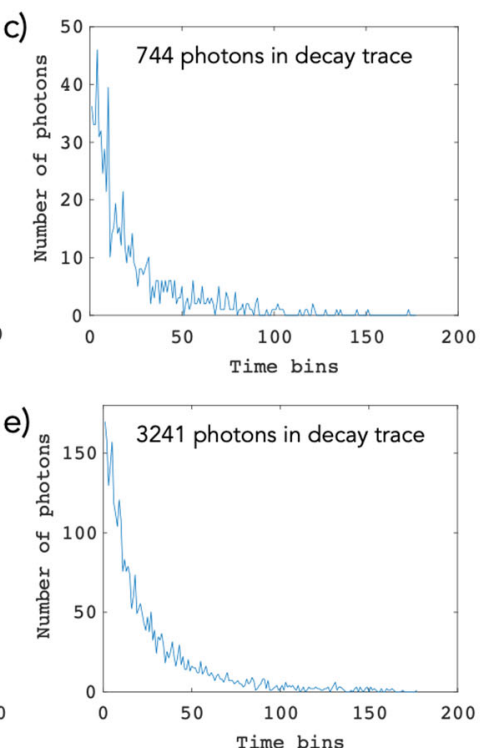

Fig. S6. The influence of cut-off values for minimum number of photons per oxygen sensor bead on standard deviations in calibration measurements. (a) Standard deviation in oxygen sensor bead population at different photon intensity cut-off values (minimum photon number per oxygen sensor bead). (b-e) Phosphorescence decay curves of oxygen sensor bead with lowest intensity in analysis with cut-off values of $300,600,1000$ and 3000 photons. Calibration measurement at $10.5 \% \mathrm{O}_{2}$ used for comparative analysis.

Two major factors influencing oxygen measurement accuracy are (i) the number of photons per bead that influence the quality of fits to phosphorescence decay curves and (ii) the oxygen tension range of the measurement, since the relation between phosphorescence lifetime and oxygen tension is non-linear. High photon numbers lead to smoother decay curves and thus more accurate fits but are negatively correlated with imaging depth and speed. In addition, given the non-linear relation of phosphorescence lifetimes and oxygen tension, in measurements at low oxygen tensions, the error in oxygen tension due to fitting errors in lifetimes is smaller compared to measurements at high oxygen tensions.

Fig. S6 shows the influence of cut-off values for minimum number of photons per oxygen sensor beads on the standard deviation in calibration measurements. This is relevant when determining the required accuracy for an experiment at hand. It is apparent in Fig. S6 that standard deviations in oxygen measurements strongly decrease when increasing the photon cut-off for oxygen sensor beads from 300 to 600 photons, while flattening off towards photon cut-off values of 1000 and 3000 photons per bead. Fig. S6b-e show decay traces from beads with the lowest intensities in a measurement with cut-off values of 300,600, 1000 and 3000, respectively, illustrating the underlying phosphorescence decay profiles used to extract phosphorescence lifetime. For typical measurements, a cut-off value of 1000 photons per bead was used in this work. However, depending on the oxygen tension range of interest and required accuracy, this can be increased or decreased. 


\section{From 3D CAD models to oxygen distribution profiles}

The microfluidic chips were designed in Inventor 2018 (Autodesk). The chip designs included flow distribution volumes at the entry to and exit from the microfluidic channel(s) of the 1-channel or 8channel systems, as well as cylindrical holes (inner diameter $0.70 \mathrm{~mm}$ ) at the chip inlet and outlet to enable stable fluidic connection to an external fluidic pump by insertion of slightly larger (outer diameter $0.80 \mathrm{~mm}$ ) blunted hypodermic needles (Fig. S7a Cross-Section).

Oxygen transport within the cell culture volume was numerically modeled using COMSOL 5.5 (COMSOL AB). Cross-sections of the two modeled geometries, matching the design channel dimensions of the physical chip, are shown in Fig. S7b, and the cross-sections were axially extruded to the length of the designed channels within the confines of the cell culture volume. Oxygen is transported into the model by convective flow (specified average flow rate) with an entry boundary condition of the equilibrium concentration for oxygen in water at $37{ }^{\circ} \mathrm{C}$ (ambient of $95 \%$ air $/ 5 \% \mathrm{CO}_{2}$, ionic strength of $170 \mathrm{mM}$ ). ${ }^{1}$ The oxygen diffusion rate in all modeled volumes (medium, channel wall, culture volume) is approximated with the value of oxygen diffusion in pure water at $37{ }^{\circ} \mathrm{C}$. Oxygen consumption in the cell culture volume is modeled by a Michaelis-Menten model using a Michaelis constant $K_{M}=4 \mu \mathrm{M}^{2}$ and varying $V_{\max }$ to match the observed oxygen distribution.

3D CAD Models
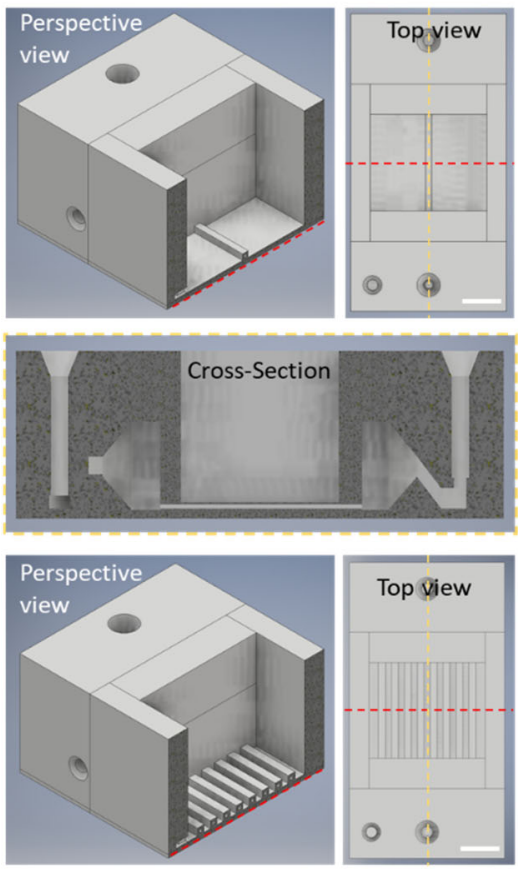

COMSOL model cross-section

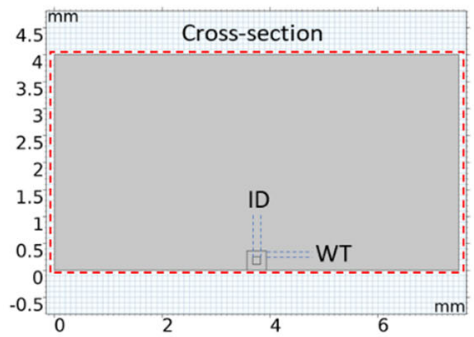

Channel inner width \& height ID $=140 \mu \mathrm{m}$ Wall thickness WT $=110 \mu \mathrm{m}$

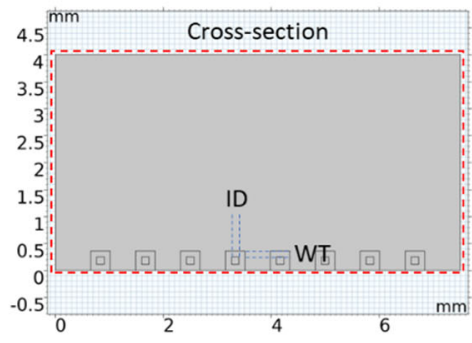

COMSOL simulation results

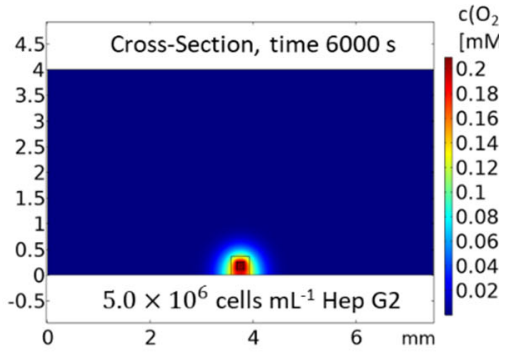

Diffusion coefficient $D\left(\mathrm{O}_{2}\right)=3.2 \times 10^{-9} \mathrm{~m}^{2} \mathrm{~s}^{-1}$ Inflow oxygen concentration $c\left(\mathrm{O}_{2}\right)=200 \mu \mathrm{M}$ Michaelis constant: $K_{\mathrm{M}}=4 \mu \mathrm{M}$

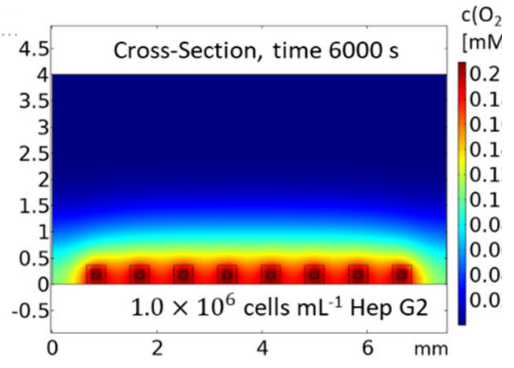

Fig. S7. Simulation of medium perfusion, oxygen diffusion and cellular oxygen consumption in microperfused cell culture scaffolds. In (a) 3D CAD models of 1-channel and 8-channel synthetic vasculature chips in areal; top and cross-sectional views are depicted. In (b) simplified 2D COMSOL models of crosssection through 3D CAD models (red dashed line) shown. In (c), simulation of oxygen distribution in 1channel and 8-channel models with perfusion, oxygen diffusion and cellular oxygen consumption are shown. Scale bar (a, top view) $2.5 \mathrm{~mm}$. 


\section{Comparison of flow velocity-dependent oxygen gradients in simulations and measurements}
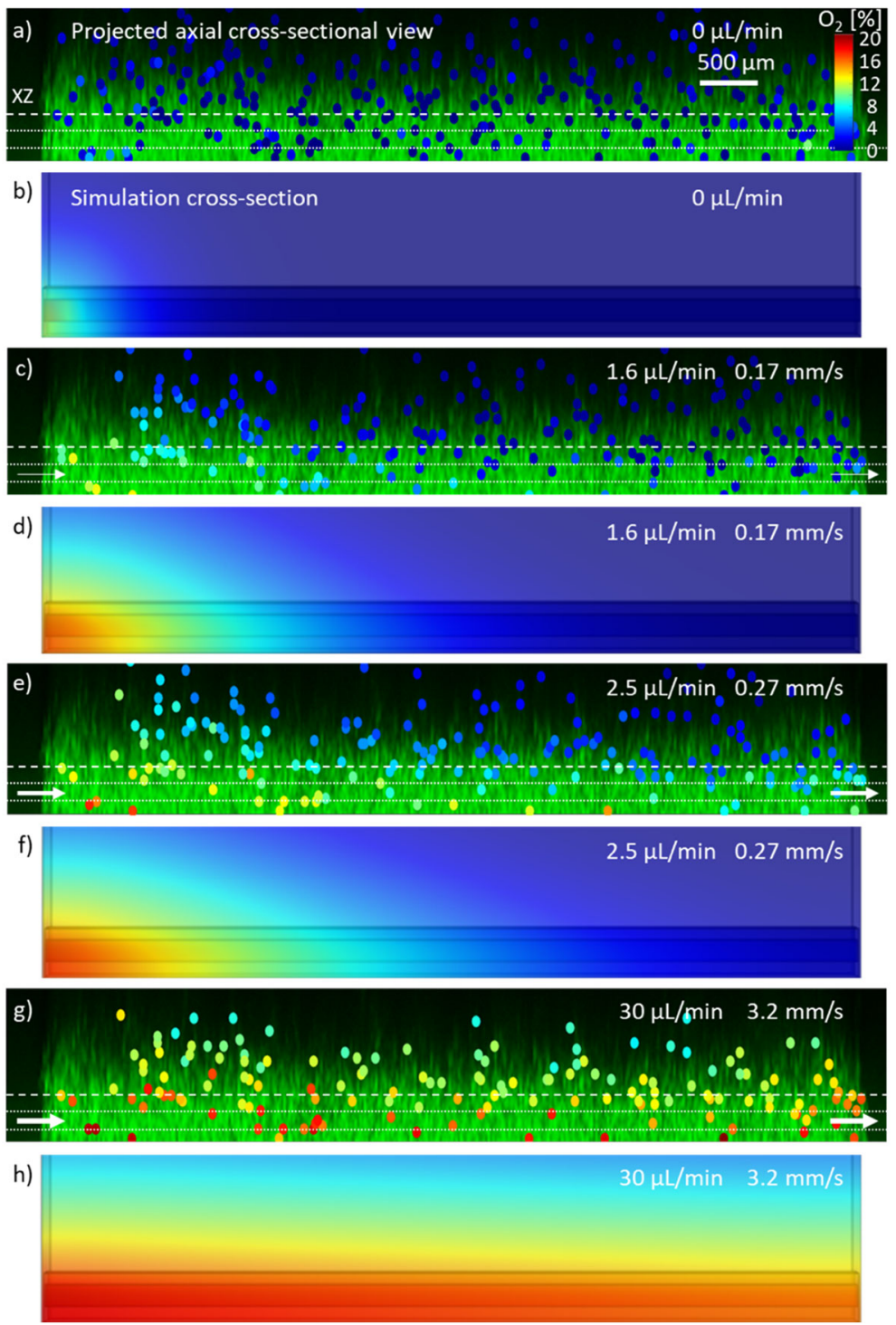

Fig. S8. Comparison of measured and numerically modeled axial oxygen concentration profiles for different perfusion rates in the 8-channel chip design at average channel flow velocities of $(a$ and $b) 0 \mathrm{~mm} / \mathrm{s}$, (c and d) $0.17 \mathrm{~mm} / \mathrm{s}$, (e and f) $0.27 \mathrm{~mm} / \mathrm{s}$, and ( $g$ and h) $3.2 \mathrm{~mm} / \mathrm{s}$. The experimental and numerically simulated seeding density is $2 \times 10^{6}$ cells $/ \mathrm{mL}$ at all flow conditions. 


\section{References for Electronic Supporting Information}

1 B. A. Wagner, S. Venkataraman and G. R. Buettner, Free Radic. Biol. Med., 2011, 51, 700-712.

2 C. Magliaro, G. Mattei, F. lacoangeli, A. Corti, V. Piemonte and A. Ahluwalia, Front. Bioeng. Biotechnol., 2019, 7, 1-9. 